\title{
A weighted de Rham operator acting on arbitrary tensor fields and their local potentials.
}

\author{
S. Brian Edgar ${ }^{1}$ and José M.M. Senovilla ${ }^{2}$ \\ ${ }^{1}$ Matematiska institutionen, Linköpings universitet \\ Linköping, Sweden S-581 83 \\ 2 Física Teórica, Universidad del País Vasco, \\ Apartado 644, 48080 Bilbao, Spain \\ e-mails: bredg@mai.liu.se, josemm.senovilla@ehu.es
}

September 26, 2018

\begin{abstract}
We introduce a weighted de Rham operator which acts on arbitrary tensor fields by considering their structure as $r$-fold forms. We can thereby define associated superpotentials for all tensor fields in all dimensions and, from any of these superpotentials, we deduce in a straightforward and natural manner the existence of $2 r$ potentials for any tensor field, where $r$ is its form-structure number. By specialising this result to symmetric double forms, we are able to obtain a pair of potentials for the Riemann tensor, and a single (2,3)-form potential for the Weyl tensor due to its tracelessness. This latter potential is the $n$-dimensional version of the double dual of the classical four dimensional $(2,1)$-form Lanczos potential. We also introduce a new concept of harmonic tensor fields, demonstrate that the new weighted de Rham operator has many other desirable properties and, in particular, it is the natural operator to use in the Laplace-like equation for the Riemann tensor.
\end{abstract}

Mathematics Subject Classifications: 58xxx, 53xxx

PACS numbers: 02.40.Ky, 02.40.Vh, 04.20.Cv

Key words: de Rham Laplacian, tensor-valued differential forms, local potentials, curvature tensors.

\section{Introduction}

The classical Helmholtz theorem [36] characterises any three dimensional vector field $\vec{V}$ on Euclidean space in terms of two potentials (scalar and vector respectively), and the 
proof depends on the existence of a solution to the vector version of Poisson's equation

$$
\nabla^{2} \stackrel{o}{\vec{V}}=\vec{V}
$$

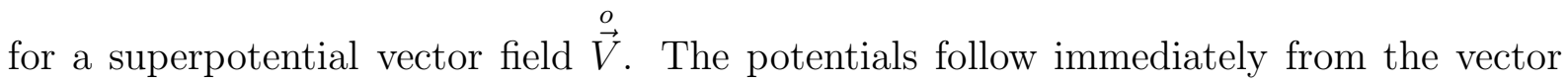
operator identity

$$
\vec{V}=\nabla^{2} \stackrel{\stackrel{o}{V}}{\equiv} \nabla(\nabla \cdot \stackrel{\stackrel{o}{\vec{V}}}{)}-\nabla \times(\nabla \times \stackrel{\stackrel{\circ}{\vec{V}}}{)} .
$$

Four dimensional [53] and $n$-dimensional generalisations [43, [32] have been discussed for vector fields using analogous arguments.

More generally, on an $n$-dimensional pseudo-Riemannian manifold, the (local) Hodge decomposition [37. (also [29, 44, 45, 33, 30, 50]) characterises any $p$-form $\Sigma$ in terms of a pair of potentials (respectively a $(p-1)$-form and a $(p+1)$-form), and the proof depends on the existence of a solution to the Laplace-like equation

$$
\Delta \stackrel{o}{\Sigma}=\Sigma
$$

for a superpotential $\Sigma^{o}$, where $\Delta \equiv d \delta+\delta d$ is the de Rham operator for $p$-forms [16], $d$ is the exterior differential and $\delta$ is the codifferential operator. The potentials follow immediately (see Result 2.2) from this definition

$$
\Sigma=\Delta \stackrel{o}{\Sigma} \equiv d(\delta \stackrel{o}{\Sigma})+\delta(d \stackrel{o}{\Sigma})
$$

In the case of general tensor fields on an $n$-dimensional pseudo-Riemannian manifold with Levi-Civita connection $\nabla$, one is still able to exploit the natural generalisations of these three operators by considering the tensor as an appropriate tensor-valued $p$-form, e.g. [5, 44, 14]. It is important to note that when a tensor (with more than $p$ antisymmetrical indices) is considered as a tensor-valued $p$-form, the generalised de Rham operator acting on such a tensor does not commute with properties involving indices from both its $p$-form part and its tensor-valued part; hence simple natural constructions for a superpotential and potentials for a tensor-valued $p$-form cannot be deduced in an analogous manner as for single $p$-forms.

Recognising this deficiency, Lichnerowicz [1, 42] proposed yet another generalisation $\Delta_{L}$ which, when acting on arbitrary tensors, commutes with all their index properties; unfortunately, the Lichnerowicz operator $\Delta_{L}$ does not have direct links to the exterior differential $d$ and codifferential operator $\delta$, and hence to natural potentials in the manner of $\Delta=d \delta+\delta d$ for single $p$-forms.

In this paper we overcome all these deficiencies in a different manner by exploiting the fact that any tensor can be considered, in a precise and unequivocal way, as an $r$-fold form [49], and defining a new generalised Laplacian which is in effect a weighted de Rham operator $\bar{\Delta}$; this operator is directly suited to the $r$-fold form structure of the given tensor and commutes with all index properties, but crucially also has direct links with appropriate exterior differential $d_{(i)}$ and codifferential $\delta_{(i)}$ operators (see Section 3). Using the new 
operator $\bar{\Delta}$ we are able to identify associated superpotentials for any tensor directly via a simple Laplace-like equation. Furthermore, we obtain, in an analogous manner, the natural generalisation of the Helmholtz and Hodge decomposition: in arbitrary curved spaces, when an arbitrary tensor is considered as an $r$-fold form then it can be expressed in a very natural and useful manner in terms of $2 r$ local potentials. By specialising these results to double forms, we can then deduce the potential structure of Riemann and Weyl curvature tensors respectively, and hence re-establish our recent result which obtained one double $(2,3)$-form potential for the Weyl tensor in all dimensions [27].

We begin in Section 2 by formulating familiar results for $p$-forms in a manner from which we can generalise to more general tensors, and in Section 3 we first review how to consider any tensor $T$ as an $r$-fold form [49. Associated with each block, $i=1,2, \ldots r$ of an $r$-fold form $T$ we have the three generalised operators $d_{(i)}, \delta_{(i)}, \Delta_{(i)}$ from which we are able to determine a superpotential and a pair of potentials; but these superpotentials and potentials are highly non-unique with limited practical use. With the introduction of the weighted de Rham operator

$$
\bar{\Delta} \equiv \frac{1}{r}\left(\Delta_{(1)}+\Delta_{(2)}+\ldots \Delta_{r}\right)=\frac{1}{r} \sum_{i=1}^{r} \Delta_{(i)}
$$

in Section 4, we establish our basic result that for an $r$-fold form $T$ there exists an associated local superpotential $\stackrel{\circ}{T}$ given by

$$
\bar{\Delta} \stackrel{o}{T}=T
$$

where it is important to note that this superpotential $\stackrel{o}{T}$ does not just have the same form structure, but also has the same index symmetry and trace properties as $T$. From this we can deduce, in a natural and straightforward manner, a generalisation of the Hodge decomposition, demonstrating the existence of $2 r$ local potentials,

$$
T=\bar{\Delta} \stackrel{o}{T}=\frac{1}{r} \sum_{i=1}^{r} \Delta_{(i)} \stackrel{o}{T} \equiv \frac{1}{r} \sum_{i=1}^{r}\left[\delta_{(i)}\left(d_{(i)} \stackrel{o}{T}\right)+d_{(i)}\left(\delta_{(i)} \stackrel{o}{T}\right)\right] .
$$

This leads naturally to a new definition for harmonic tensors, in general.

In Section 5 we specialise these results to double $(q, p)$-forms and identify their four different potentials. Further specialisations are made in Section 6: first to (anti)symmetric double $(p, p)$-forms, which are shown to have only a pair of potentials - a double $(p, p+1)$ form and a double $(p, p-1)$-form - and then to the particular value $p=2$. These results are immediately applicable to the Riemann curvature tensor-Theorem 6.3 - and to the traceless Weyl tensor - Theorem 6.4, [27].

In Section 7 we demonstrate that the new weighted de Rham operator is the natural operator to use in the Laplace-like equation for the Riemann tensor, and we illustrate its advantages over the particular de Rham operator which is usually used. In Section 8 we summarise, demonstrate how this work provides insights into some other investigations, and outline future plans. 


\section{Standard results for $p$-forms.}

Let $V_{n}$ be any differentiable $n$-dimensional pseudo-Riemannian manifold endowed with a metric $g_{a b}$ of arbitrary signature. The covariant derivative associated with $g$ is denoted either by $\nabla$ or by a semicolon, e.g., $\nabla_{a} v_{b} \equiv v_{b ; a}$; and its Riemann and Ricci tensors by $R_{a b c d}$ and $R_{a b} \equiv R_{a c b}^{c}$, respectively. Our convention for the Riemann tensor follows from the Ricci identity:

$$
2 v_{a ;[b c]}=R_{a b c}^{d} v_{d} .
$$

As usual, we use round and square brackets to indicate symmetrization and antisymmetrization of indices, respectively.

The graded algebra of exterior forms is $\Lambda$, with $\Lambda^{p}$ denoting the set of exterior $p$-forms; in particular, for $p=0$, the elements of $\Lambda^{0}$ will be scalar functions, (also called sometimes 0 -forms). The canonical volume element $n$-form $\eta \in \Lambda^{n}$ is denoted by $\eta_{a_{1} \ldots a_{n}}=\eta_{\left[a_{1} \ldots a_{n}\right]}$. Then, we can define the standard Hodge dual operator $*: \Lambda^{p} \longrightarrow \Lambda^{n-p}$ by means of

$$
{\stackrel{\Sigma}{a_{p+1} \ldots a_{n}}}^{*} \equiv \frac{1}{p !} \eta_{a_{1} \ldots a_{n}} \Sigma^{a_{1} \ldots a_{p}} \quad \forall \Sigma \in \Lambda^{p} .
$$

It is easy to prove that $* *=\epsilon(-1)^{p(n-p)}$ where $\epsilon= \pm 1=\operatorname{sign}\left(\operatorname{det}\left(g_{a b}\right)\right)$ is a sign depending on the signature.

One can define a scalar product $<,>$ on each $\Lambda^{p}$ in the standard way by first defining the function

$$
(\Sigma, \Phi) \equiv \Sigma_{a_{1} \ldots a_{p}} \Phi^{a_{1} \ldots a_{p}}
$$

for every $\Sigma, \Phi \in \Lambda^{p}$ and then integrating this over the manifold $V_{n}$

$$
<\Sigma, \Phi>\equiv \int_{V_{n}}(\Sigma, \Phi) \eta=p ! \int_{V_{n}} \Sigma \wedge \stackrel{*}{\Phi}
$$

When $V_{n}$ is compact without boundary the integration can be carried out without further restrictions; otherwise, at least one of $\Sigma, \Phi$ has to be assumed to have compact support. This will be assumed without explicit mention in what follows. This scalar product is bi-linear, symmetric and non-degenerate. In the case of proper Riemannian manifolds, it is also positive definite.

The standard operations on the exterior algebra are the exterior differential (also called 'curl') $d: \Lambda^{p} \longrightarrow \Lambda^{p+1}$ and codifferential (also called 'divergence') $\delta: \Lambda^{p} \longrightarrow \Lambda^{p-1}$ [11, 42, 29, 6, 44, 51]. The second is simply defined by

$$
\delta \equiv(-1)^{p} *^{-1} d *=\epsilon(-1)^{(n-p)(p-1)+1} * d *
$$

when acting on $p$-forms. With index notation they can be given as

$$
\begin{gathered}
(d \Sigma)_{a_{1} \ldots a_{p+1}} \equiv(p+1) \nabla_{\left[a_{1}\right.} \Sigma_{\left.a_{2} \ldots a_{p+1}\right]}=(-1)^{p}(p+1) \Sigma_{\left[a_{1} \ldots a_{p} ; a_{p+1}\right]} \\
(\delta \Sigma)_{a_{2} \ldots a_{p}} \equiv-\nabla^{a_{1}} \Sigma_{a_{1} a_{2} \ldots a_{p}}=(-1)^{p} \Sigma_{a_{2} \ldots a_{p} a_{1}} ; a_{1}
\end{gathered}
$$

for all $\Sigma \in \Lambda^{p}$. Observe that $d \alpha=0, \forall \alpha \in \Lambda^{n}$ and, as is implicit in this formula and follows trivially from the definition (3) of $\delta$, for functions $f$ one has: $\delta f=0, \forall f \in \Lambda^{0}$. 
Elementary properties of these operators are $d^{2} \equiv 0$ and $\delta^{2} \equiv 0$. Moreover, for every $\Sigma \in \Lambda^{p}$ and $\Gamma \in \Lambda^{p+1}$ we have

$$
<d \Sigma, \Gamma>=<\Sigma, \delta \Gamma>
$$

as can be easily checked by using the Gauss theorem. Therefore, $d$ and $\delta$ are mutually adjoint with respect to $<,>$ 41, 5]. As usual, this property can be used to define $d$ and $\delta$ acting on $p$-form distributions by means of the standard use of "test $p$-forms" (i.e., $C^{\infty}$ $p$-forms of compact support), see [41.

A $p$-form $\Sigma$ is called closed if $d \Sigma=0$ and exact if $\Sigma=d \Psi$ for some $\Psi \in \Lambda^{p-1}$. Analogously $\Sigma$ is said to be co-closed if $\delta \Sigma=0$ and co-exact if $\Sigma=\delta \Gamma$ for some $\Gamma \in \Lambda^{p+1}$. In general, every statement on $p$-forms has a dual statement replacing $d$ for $\delta$ and the form by its Hodge dual. Obviously, all exact $p$-forms are closed, but the converse is not true, globally, in general. The Poincaré lemma ensures that every closed form is locally exact. Analogously, all co-exact forms are co-closed, and these are locally co-exact.

The de Rham cohomology class of order $p$ is defined as the quotient of the set of closed $p$-forms by the set of exact $p$-forms. And the de Rham Laplacian operator $\Delta: \Lambda^{p} \longrightarrow \Lambda^{p}$ is defined intrinsically by $\Delta \equiv d \delta+\delta d$ (sometimes also written as $\Delta=(d+\delta)^{2}$ ) [16]. Its formula with index notation is

$$
(\Delta \Sigma)_{a_{1} \ldots a_{p}}=-\nabla^{c} \nabla_{c} \Sigma_{a_{1} \ldots a_{p}}+p R_{c\left[a_{1}\right.} \Sigma_{\left.a_{2} \ldots a_{p}\right]}^{c}-\frac{p(p-1)}{2} R_{c d\left[a_{1} a_{2}\right.} \Sigma^{c d}{ }_{\left.a_{3} \ldots a_{p}\right]} .
$$

Observe that the Riemann tensor terms are not present for 1-forms, while for 0-forms $\Delta$ is (minus) the usual Laplacian $\nabla^{c} \nabla_{c}=g^{b c} \nabla_{b} \nabla_{c}$.

The following important properties of $\Delta$ are easily obtained (see e.g. [16, 41, 29, 45])

Result 2.1 The operator $\Delta$ is linear, self-adjoint with respect to $<,>$

$$
<\Delta \Sigma, \Phi>=<\Sigma, \Delta \Phi>\quad \forall \Sigma, \Phi \in \Lambda^{p}
$$

and also commutes with $*$, $d$ and $\delta$

$$
* \Delta=\Delta *, \quad d \Delta=\Delta d, \quad \delta \Delta=\Delta \delta .
$$

Furthermore, we can immediately prove the identity

$$
<\Sigma, \Delta \Sigma>=<d \Sigma, d \Sigma>+<\delta \Sigma, \delta \Sigma>
$$

In the case of proper Riemannian manifolds this implies that $\Delta$ is a positive operator, i.e., $\left\langle\Sigma, \Delta \Sigma>\geq 0\right.$ for all $\Sigma \in \Lambda^{p}$.

In what follows, and in order to keep the technical complications to a minimum, we shall assume that the metric $g$ is analytical. This will allow us to use the simpler versions of the existence results for solutions of Laplace-like equations. More powerful results could of course be used by assuming $C^{2}$ differentiability of the metric, but this will not change the main points we wish to make in this paper. Keeping this in mind, a key result for $p$-forms [29, 45, 30], which we will generalise in this paper, is 
Result 2.2 (Local Helmholtz-Hodge decomposition) Given any p-form $\Sigma \in \Lambda^{p}$, there always exists a local superpotential $\Sigma^{o} \in \Lambda^{p}$ such that $\Delta \stackrel{o}{\Sigma}=\Sigma$; furthermore there always exists a pair of local potentials $(\Psi, \Gamma)$ with $\Psi \in \Lambda^{p-1}$ and $\Gamma \in \Lambda^{p+1}$ such that

$$
\Sigma=d \Psi+\delta \Gamma
$$

where $\Psi=\delta \stackrel{o}{\Sigma}$ and $\Gamma=d \stackrel{o}{\Sigma}$.

Proof. From the structure of the de Rham operator $\Delta$ in (44), according to the Cauchy-

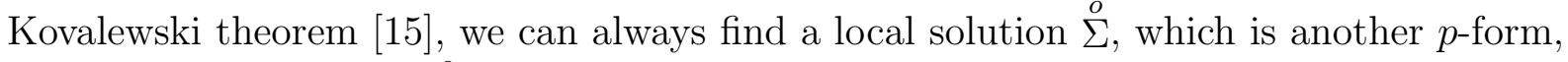
to the equation $\Sigma=\Delta \stackrel{o}{\Sigma}$. The remainder of the result then follows from the definition $\Delta \equiv d \delta+\delta d$.

Note that $\Psi$ is missing for the case $p=0$ so that in this case the statement is just that any function is locally the divergence of a vector field, while for $p=n, \Gamma$ is identically zero stating that any $n$-form is closed ergo locally exact.

An index version of Result 2.2 is

$$
\begin{aligned}
\left.(\Delta)^{o}\right)_{a_{1} \ldots a_{p}} & \left.=(-1)^{p} p \Sigma_{c\left[a_{1} \ldots a_{p-1}\right.} ; a_{p}\right]-(p+1) \sum_{\left[a_{1} \ldots a_{p} ; c\right]}^{c} \\
& =(-1)^{p-1} p \Psi_{\left[a_{1} \ldots a_{p-1} ; a_{p}\right]}-\Gamma_{c a_{1} \ldots a_{p}} ;{ }^{c} \\
& =(d \Psi)_{a_{1} \ldots a_{p}}+(\delta \Gamma)_{a_{1} \ldots a_{p}} \\
& =\Sigma_{a_{1} \ldots a_{p}}
\end{aligned}
$$

where we have defined

$$
\begin{aligned}
& \Psi_{a_{1} \ldots a_{p-1}} \equiv-{\stackrel{D}{c} a_{1} \ldots a_{p-1}}^{; c}, \\
& \Gamma_{a_{1} \ldots a_{p+1}} \equiv(-1)^{p}(p+1) \stackrel{o}{\Sigma}_{\left[a_{1} \ldots a_{p} ; a_{p+1}\right]} \text {. }
\end{aligned}
$$

Observe that this result is independent of any field equations for $\Sigma$, and is a local result. Actually, Result 2.2 can be strengthened in the case of compact without boundary proper Riemannian manifolds and one can obtain the global Hodge decomposition theorem [37, 16. 34, 41, 29, 45, 30, 50]: any $\Sigma \in \Lambda^{p}$ admits a unique global decomposition as $\Sigma=d \Psi+\delta \Gamma+\Upsilon$ where $\Psi \in \Lambda^{p-1}, \Gamma \in \Lambda^{p+1}$ and $\Upsilon \in \Lambda^{p}$ is a harmonic $p$-form (that is, $\Upsilon$ is closed and co-closed, i.e., $\Delta \Upsilon=0$ ) in the same co-homology class as $\Sigma$. Note that the global Hodge result can be used to obtain the more modest local Result 2.2 because, since $\Upsilon$ is closed and co-closed, it is also locally exact and co-exact (see 34]).

Closely linked to the existence of potentials is their gauge freedom, and there are well known results for $p$-forms which exploit this gauge freedom. However, when one considers the more complicated tensor-valued forms which are the subject of this paper, there are aspects of the role of gauge which are significantly different from the $p$-form results. A detailed discussion of the role and application of gauge for tensor valued forms will be presented in 28 . 


\section{Generalization to arbitrary tensors: $r$-fold forms}

In the previous section the two crucial results were being able to identify a superpotential via a Laplace-like equation for the de Rham operator, and then being able to link potentials in a simple natural manner to derivatives of the superpotential, via this de Rham operator, which is of course defined only for differential forms. The question now is how to generalize all this to arbitrary tensors.

Long ago Lichnerowicz [41, 42 proposed a generalised Laplacian for arbitrary tensor fields which had the first of these properties, as well as a number of other useful properties. Given an arbitrary rank- $m$ tensor field $T_{a_{1} \ldots a_{m}}$, the Lichnerowicz operator $\Delta_{L}$ can be defined as

$$
\begin{aligned}
\left(\Delta_{L} T\right)_{a_{1} \ldots a_{m}} \equiv-\nabla^{c} \nabla_{c} T_{a_{1} \ldots a_{m}}+ & \sum_{s=1}^{m} R_{a_{s}}^{c} T_{a_{1} \ldots a_{s-1} c a_{s+1} \ldots a_{m}} \\
& -\sum_{s \neq t}^{m} R_{a_{s}{ }^{d} a_{t}}^{c} T_{a_{1} \ldots a_{s-1} c a_{s+1} \ldots a_{t-1} d a_{t+1} \ldots a_{m}} .
\end{aligned}
$$

This operator has a number of very important properties 41, 42]:

- $\Delta_{L}$ respects the symmetry properties of $T_{a_{1} \ldots a_{m}}$; that is to say, $\left(\Delta_{L} T\right)_{a_{1} \ldots a_{m}}$ has exactly the same index symmetries as $T_{a_{1} \ldots a_{m}}$.

- $\Delta_{L}$ commutes with traces, i.e., the trace on any two indices of $\left(\Delta_{L} T\right)_{a_{1} \ldots a_{m}}$ equals $\Delta_{L}$ applied to the corresponding trace of $T_{a_{1} \ldots a_{m}}$; in particular, if $T_{a_{1} \ldots a_{m}}$ is traceless in any pair of indices, then so is $\left(\Delta_{L} T\right)_{a_{1} \ldots a_{m}}$.

- $\Delta_{L}$ is self-adjoint with respect to the scalar product $\{$,$\} defined by$

$$
\{T, S\} \equiv \int_{V_{n}} T_{a_{1} \ldots a_{m}} S^{a_{1} \ldots a_{m}} \eta
$$

for arbitrary $T, S \in T_{m}\left(V_{n}\right)$. (Of course, the same comments as followed (2) on the compactness of $V_{n}$ or of the support of one of the tensors, are in order here.) Therefore $\left\{\Delta_{L} T, S\right\}=\left\{T, \Delta_{L} S\right\}$.

- In manifolds with a parallel Ricci tensor $\left(\nabla_{a} R_{b c}=0\right.$, which includes the important cases of Einstein spaces and Ricci-flat manifolds,) the following two properties hold; see [41, 42]:

- when acting on rank-1 tensors, $\Delta_{L}$ commutes with the covariant derivative;

- when acting on rank-2 tensors, $\Delta_{L}$ commutes with the divergence operator.

- When acting on $p$-forms, $\Delta_{L}$ coincides with the de Rham Laplacian $\Delta$ :

$$
\Delta_{L} \Sigma=\Delta \Sigma, \quad \forall \Sigma \in \Lambda^{p}
$$


Unfortunately, the Lichnerowicz Laplacian $\Delta_{L}$ does not have simple natural links to first derivative operators for arbitrary tensors in the same way as the de Rham operator has for $p$-forms.

We believe that the best way to extend the principles of Section 2 to arbitrary tensors is to consider tensors as $r$-fold forms. This terminology was extensively considered in [49, and the underlying simple idea is based on the following remark: Given any rank- $m$ tensor $T^{a_{1} \ldots a_{m}}$, there is a minimum natural number $r, r \leq m$ and a unique set of $r$ natural numbers $n_{1}, \ldots, n_{r}$, with $\sum_{i=1}^{r} n_{i}=m$, such that $T^{a_{1} \ldots a_{m}}$ is a linear map on $\Lambda^{n_{1}} \times \ldots \times \Lambda^{n_{r}}$. In other words, there always exists a minimum $r$ such that $\tilde{T} \in \Lambda_{n_{1}} \otimes \ldots \otimes \Lambda_{n_{r}}$, where $\tilde{T}^{a_{1} \ldots a_{m}}$ is the appropriate permuted version of $T^{a_{1} \ldots a_{m}}$ which selects the natural order for the $n_{1}, \ldots, n_{r}$ entries. Tensors seen in this way are called $r$-fold $\left(n_{1}, \ldots, n_{r}\right)$-forms [49]. In short, all tensors can be considered, in a precise way, as $r$-fold forms.

Definition 3.1 (Form-structure number and block ranks) For any tensor $T$, the uniquely defined number $r$ will be called its form-structure number, and each of the $n_{i}$, the $i$-th block rank.

Some simple examples are: any $p$-form $\Sigma$ is trivially a single (that is, 1 -fold) $p$-form, while $\nabla \Sigma$ is a double $(1, p)$-form, with $r=2$ and $n_{1}=1, n_{2}=p$; the Riemann tensor $\left(r=2, n_{1}=n_{2}=2\right)$ is a double (2,2)-form which is symmetric (the pairs can be interchanged); the Ricci tensor is a double symmetric (1,1)-form and, in general, any completely symmetric rank- $r$ tensor is an $r$-fold $(1,1, \ldots, 1)$-form. A 3-tensor $A_{a b c}$ with the property $A_{a b c}=-A_{c b a}$ is a double (2,1)-form and the corresponding $\tilde{A}$ is clearly given by $\tilde{A}_{a b c}=\tilde{A}_{[a b] c} \equiv A_{a c b}$. The standard index version of familiar tensors such as Riemann tensors $R_{a b c d}=R_{[a b][c d]}$, Weyl tensors $C_{a b c d}=C_{[a b][c d]}$, torsion tensors $T_{a b c}=T_{[a b] c}$, or Lanczos tensors $H_{a b c}=H_{[a b] c}$, already have the indices in the appropriate permuted version, so that they coincide with their tilded versions. In these cases we shall dispense with the label.

The scalar product (22) can be immediately generalized to arbitrary tensors of the same type (i.e., with the same form-structure number $r$ and block ranks) by first defining

$$
(T, S) \equiv \tilde{T}_{a_{1} \ldots a_{m}} \tilde{S}^{a_{1} \ldots a_{m}}
$$

and then (same comments as before on compactness)

$$
<T, S>\equiv \int_{V_{n}}(T, S) \eta .
$$

Observe that this scalar product is adapted to the structure as $r$-fold forms of the tensor fields $T$ and $S$, and therefore it is different from the product defined in (13), so that we have, in general, $\langle T, S>=\{\tilde{T}, \tilde{S}\} \neq\{T, S\}$. This will be relevant in what follows. Note, moreover, that $\{$,$\} is defined for general tensors T, S$, not only for those with the same form-structure number $r$ and block ranks. As before, the product (14) is bi-linear, symmetric and non-degenerate. For proper Riemannian manifolds, it is also positive definite. 
Now consider any tensor field $T$ and let $r$ be its form-structure number. For each one of the $r$ antisymmetric blocks, one can follow a procedure similar to that recalled above for $p$-forms. To fix ideas, let us select the $i$-th block, with block rank $n_{i}$, to treat $T$ as a tensor-valued $n_{i}$-form, so that $\left.\tilde{T}^{a_{1} \ldots a_{h}}{ }_{b_{1} \ldots b_{n_{i}}}{ }^{a_{h+1} \ldots a_{k}}=\tilde{T}^{a_{1} \ldots a_{h}}{ }_{\left[b_{1} \ldots b_{n_{i}}\right]}\right]^{a_{h+1} \ldots a_{k}}$ with a set of $n_{i} \leq n$ completely antisymmetrical indices $b_{1} \ldots b_{n_{i}}$ plus a number of extra indices denoted by $a_{1} \ldots a_{h}$ and $a_{h+1} \ldots a_{k}$. These objects are usually referred to as differential tensor forms or tensor-valued differential forms, e.g. [11, 5, 44, 14. Then, the previous definition (11) can be trivially extended so that the dual of $T$ with respect to the $i$-th block $b_{1} \ldots b_{n_{i}}$ is denoted as $*_{(i)} T$ and defined by [49]

$$
\left(*_{(i)} T\right)^{a_{1} \ldots a_{h}}{ }_{b_{n_{i}+1} \ldots b_{n}}{ }^{a_{h+1} \ldots a_{k}} \equiv \frac{1}{n_{i} !} \eta_{b_{1} \ldots b_{n}} \tilde{T}^{a_{1} \ldots a_{h} b_{1} \ldots b_{n_{i}} a_{h+1} \ldots a_{k}}
$$

As before, $*_{(i)} *_{(i)}=\epsilon(-1)^{n_{i}\left(n-n_{i}\right)}$ when acting on $\tilde{T}$.

Next we extend the definitions of $d, \delta, \Delta$ from the previous section to differential $d_{(i)}$, co-differential $\delta_{(i)}$, and de Rham operator $\Delta_{(i)}$ acting on the $i$-th block of the $r$-fold form T as: ${ }^{1}$

$$
\begin{aligned}
\left(d_{(i)} T\right)^{a_{1} \ldots a_{h}}{ }_{b_{1} \ldots b_{n_{i}+1}}^{a_{h+1} \ldots a_{k}} & \equiv(-1)^{n_{i}}\left(n_{i}+1\right) \tilde{T}^{a_{1} \ldots a_{h}}{ }_{\left[b_{1} \ldots b_{n_{i}}{ }^{a_{h+1} \ldots a_{k}}{ }_{\left.; b_{n_{i}+1}\right]}\right.}, \\
\delta_{(i)}=(-1)^{n_{i}} *_{(i)}^{-1} d_{(i)} *_{(i)} & \equiv \epsilon(-1)^{\left(n-n_{i}\right)\left(n_{i}-1\right)+1} *_{(i)} d_{(i)} *_{(i)} \\
\Delta_{(i)} & \equiv d_{(i)} \delta_{(i)}+\delta_{(i)} d_{(i)} .
\end{aligned}
$$

(An alternative equivalent definition for $\delta_{(i)}$ can be given via $d_{\left(i^{\prime}\right)}$ and a contraction (trace) operator across the $i$-th and $i^{\prime}$-th blocks [39]; see comments following the definition of the trace operator in Section 5.)

We emphasise that the covariant derivative acts on all indices, i.e., the extra tensor indices as well as the explicit form indices. Although the definition for $d$ was extended to a tensor valued form in [11] and is well known, as far as we are aware all three operators, in the form given above, were first introduced in [5], and are less familiar. In this more general context, sometimes the extended $d$ is called 'the absolute exterior differential' or 'the covariant exterior differential'; some references retain $d$ and $\delta$ [44], some texts replace $d(\delta, \Delta)$ with $\tilde{d}(\tilde{\delta}, \tilde{\Delta})[5]$, or with $d^{\nabla}\left(\delta^{\nabla}, \Delta^{\nabla}\right)[7$, 14, 52], or with $D$ [11, 39, 151, 6, 33, 45, 8, 9], or with $\nabla$ [30]. We shall use the notation of (16], 17, 18) since we wish to be able to combine different de Rham operators.

The operator $d_{(i)}\left(\delta_{(i)}\right)$ produces another tensor with one more (one less) index in general, and this new tensor has in general the same form-structure number $r$. Of course, as for single $p$-forms, there are some special situations: (i) if $n_{i}=n$ then $d_{(i)} T=0$; (ii) for any tensor $T$ with form structure number $r$, $\nabla T$ has form-structure number $r+1$ and can be considered as a definition of ' $d_{(r+1)} T$ '; (iii) if $n_{i}=1$ then $\delta_{(i)} T$ has $r-1$ as form-structure number. In this case, in order to compute $\Delta_{(i)}$ one has to allow the operator $d_{(i)}$ in the combination $d_{(i)} \delta_{(i)}$ to act on the missing block - as if $\delta_{(i)} T$ were an $r$-fold $\left(n_{1}, \ldots, n_{i-1}, 0, n_{i+1} \ldots n_{r}\right)$-form - in the same way as in (ii).

\footnotetext{
${ }^{1}$ When applying the operators $*_{(i)}, d_{(i)}, \delta_{(i)}, \Delta_{(i)}$ we are defining the resulting tensor as having the $i$-th block in the ordered position, so that $\widetilde{*_{(i)} T}=*_{(i)} T, \widetilde{d_{(i)} T}=d_{(i)} T$, etc.
} 
Taking this into account, we need definitions for $d_{(\ell)} T, \delta_{(\ell)} T$ when $\ell \neq 1, \ldots, r$, which are simply

$$
d_{(\ell)} T=\widehat{\nabla \tilde{T}}, \quad \delta_{(\ell)} T=0, \quad \ell \notin\{1, \ldots, r\}
$$

where in the first case the - means that the extra index provided by the covariant derivative must be placed in the appropriate place within $\{1, \ldots, r+1\}$.

Bearing all this in mind, we can write down explicit index formulas for $\delta_{(i)}, \Delta_{(\ell)}$ and $\Delta_{(i)}$ for the cases $i \in\{1, \ldots, r\} \not \ngtr \ell$ :

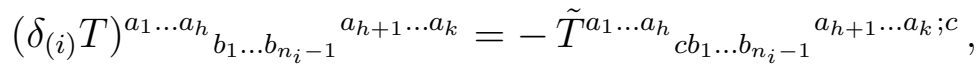

$$
\begin{aligned}
& \left(\Delta_{(\ell)} T\right)_{a_{1} \ldots a_{m}}=-\nabla^{c} \nabla_{c} \tilde{T}_{a_{1} \ldots a_{m}} \\
& \left(\Delta_{(i)} T\right)^{a_{1} \ldots a_{h}} b_{1} \ldots b_{n_{i}}{ }^{a_{h+1} \ldots a_{k}}=-\nabla^{c} \nabla_{c} \tilde{T}^{a_{1} \ldots a_{h}} b_{b_{1} \ldots b_{n_{i}}}{ }^{a_{h+1} \ldots a_{k}} \\
& +n_{i} R_{c\left[b_{1}\right.} \tilde{T}_{\left.b_{2} \ldots b_{n_{i}}\right]^{a_{1} \ldots a_{h+1} \ldots a_{k}}}^{a_{h}}-\frac{n_{i}\left(n_{i}-1\right)}{2} R_{c d\left[b_{1} b_{2} b\right.} \tilde{T}_{\left.a_{3} \ldots b_{n_{i}}\right]}^{a_{h+1} \ldots a_{h} c a_{k}} \\
& -n_{i} \sum_{s=1}^{k} R_{c}^{a_{i}} d\left[b_{1} \tilde{T}^{a_{s} \ldots a_{s-1} c a_{s+1} \ldots a_{h} d} b_{\left.b_{2} \ldots b_{n_{i}}\right]} a_{h+1} \ldots a_{k} .\right.
\end{aligned}
$$

A trivial calculation using the Gauss theorem leads to

$$
<d_{(i)} T, U>=<T, \delta_{(i)} U>
$$

where $U$ and $T$ have the same form-structure number $r$ and $n_{i}(T)+1=n_{i}(U)$. Thus, the operators $d_{(i)}$ and $\delta_{(i)}$ are adjoint to each other with respect to $<,>$ introduced in (14).

Observe the similarities and the differences of $\Delta_{(i)}$ of (22) with $\Delta_{L}$ as defined in (13):

- For each $i=1, \ldots, r, \Delta_{(i)} T$ respects the skew-symmetry on the $i$-th antisymmetric block of $T$, and the symmetries and trace properties on the extra indices not in that block; this implies, in particular, that $\Delta_{(i)} T$ has the same form-structure number and block ranks as $T$.

- On the other hand, any mixed trace, or mixed index symmetry, involving indices from both the explicit $i$-th antisymmetric block and the rest of the indices is not preserved in general.

- For each $i=1, \ldots, r, \Delta_{(i)}$ is self-adjoint with respect to the scalar product (14), so that $\left\langle\Delta_{(i)} T, S\right\rangle=<T, \Delta_{(i)} S>$ for all $T, S$ with the same form-structure number and block ranks. Moreover, one can prove the identities

$$
<T, \Delta_{(i)} T>=<d_{(i)} T, d_{(i)} T>+<\delta_{(i)} T, \delta_{(i)} T>\quad \forall i \in\{1, \ldots, r\}
$$

in the same way as (17) was obtained. From here, in particular, one deduces that $\Delta_{(i)}$ are positive operators in proper Riemannian manifolds: $\left\langle T, \Delta_{(i)} T\right\rangle \geq 0$.

- When $r=1$, that is to say, when acting on $p$-forms, $\Delta_{(1)}$ coincides with the de Rham operator: $\Delta_{(1)} \Sigma=\Delta \Sigma=\Delta_{L} \Sigma$ for all $\Sigma \in \Lambda^{p}$. In particular, when acting 
on rank-1 tensors, $\Delta_{(1)}$ commutes with the covariant derivative in manifolds with a parallel Ricci tensor $\left(\nabla_{a} R_{a b}=0\right)$. There is however no simple commutation relation between $\Delta_{(i)}$ and $\delta_{(i)}$; see (26) below.

As with $p$-forms, we can put forward the following definition:

Definition 3.2 ( $i$-harmonic and fully harmonic tensors) A tensor field $T$ with formstructure number $r$ is said to be i-harmonic, for $i \in\{1, \ldots, r\}$, if and only if $\Delta_{(i)} T=0$. Such a tensor will be called fully harmonic if it is $i$-harmonic for all $i=1, \ldots, r$.

Note that the harmonic property in the sense of Lichnerowicz (i.e., $\Delta_{L} T=0$ ) is different from these new harmonic properties. This may have important consequences for the theory of harmonic tensors in Riemannian or pseudo-Riemannian manifolds. See Definition 4.1 and the results which follow it.

It is important to note, unlike the situation for $p$-forms, that $d_{(i)}^{2} \neq 0$ and $\delta_{(i)}^{2} \neq 0$ in curved spaces. Using the Ricci identity, one obtains

$$
\begin{aligned}
& \left(d_{(i)}^{2} T\right)^{a_{1} \ldots a_{h}} b_{1} \ldots b_{n_{i}+2}{ }^{a_{h+1} \ldots a_{k}} \\
& \left.=\frac{1}{2}\left(n_{i}+1\right)\left(n_{i}+2\right) \sum_{s=1}^{k} R^{a_{s}}{ }_{c b_{n_{i}+1} b_{n_{i}+2}} \tilde{T}^{a_{1} \ldots a_{s-1} c a_{s+1} \ldots a_{h}}{ }_{\left.b_{1} \ldots b_{n_{i}}\right]}\right]_{h+1}^{a_{h+1} \ldots a_{k}}, \\
& \left(\delta_{(i)}^{2} T\right)^{a_{1} \ldots a_{h}}{ }_{b_{1} \ldots b_{n_{i}-2}}^{a_{h+1} \ldots a_{k}}=-\frac{1}{2} \sum_{s=1}^{k} R_{c}^{a_{s}}{ }_{c}^{d e} \tilde{T}^{a_{1} \ldots a_{s-1} c a_{s+1} \ldots a_{h}}{ }_{d e b_{1} \ldots b_{n_{i}-2}}^{a_{h+1} \ldots a_{k}} .
\end{aligned}
$$

Note that for higher derivatives all derivatives of the Riemann tensor disappear because of the Bianchi identities; this means that even derivatives will give terms involving products of the Riemann tensor and $\tilde{T}$, while odd derivatives will give terms involving products of the Riemann tensor and a derivative of $\tilde{T}$.

have a similar product structure with the Riemann tensor, since all derivatives of the Riemann tensor disappear because of the Bianchi identities.

Observe that, in flat spaces, these operators are nilpotent. (Compare with [19, where similar ideas are developed in flat Euclidean spaces.) Note also that $d_{(i)}^{2}=0$ whenever $n_{i} \in\{n-1, n\}$, and that $\delta_{(i)}^{2}=0$ if $n_{i}=1$. More generally, $d_{(i)}^{m}=0$ for $m>n-n_{i}$, and $\delta_{(i)}^{m}=0$ for $m>n_{i}$, so these curved space operators are also nilpotent, but depending on the dimension of the space and the block rank of the tensor-valued form being acted on.

The commutation properties with $\Delta_{(i)}$ follow directly from (24) 25 using

$$
d_{(i)} \Delta_{(i)}-\Delta_{(i)} d_{(i)}=d_{(i)}^{2} \delta_{(i)}-\delta_{(i)} d_{(i)}^{2}, \quad \delta_{(i)} \Delta_{(i)}-\Delta_{(i)} \delta_{(i)}=\delta_{(i)}^{2} d_{(i)}-d_{(i)} \delta_{(i)}^{2} .
$$

(Observe that, in flat spaces, these operators commute.) Finally, $\Delta_{(i)}$ commute with each of the Hodge dual operators $*_{(j)}$ defined in (15)

$$
*_{(j)} \Delta_{(i)}=\Delta_{(i)} *(j) \quad \forall i, j \in\{1, \ldots, r\} .
$$

The usefulness of these operators (16), (17), (18) is that we can now translate the calculation performed in (9) to arbitrary tensors. Although the operators $\Delta_{(i)}$ are different from the de Rham operator $\Delta$ it is still possible to apply the Cauchy-Kovalewski theorem [15], and we can generalise Result 2.2 to 
Result 3.1 Let $T$ be any tensor field, and let $r$ be its form-structure number and $\left(n_{1}, \ldots, n_{r}\right)$ its block ranks. There always exist local superpotentials $\stackrel{\circ}{T}$ with the same form-structure number and block ranks as $T$ such that $\Delta_{(i)} \stackrel{o}{T}=T$; furthermore, for each $i=1, \ldots, r$ there always exist a pair of local potentials $\left(Y_{(i)}, Z_{(i)}\right)$, such that

$$
T=\delta_{(i)}\left(d_{(i)} \stackrel{o}{T}\right)+d_{(i)}\left(\delta_{(i)} \stackrel{o}{T}\right) \equiv \delta_{(i)} Y_{(i)}+d_{(i)} Z_{(i)}
$$

where we have defined the r-fold form potentials $Y_{(i)}=d_{(i)} \stackrel{o}{T}$ and $Z_{(i)}=\delta_{(i)} \stackrel{o}{T}$.

In index notation:

$$
\left(\Delta_{(i)} \stackrel{o}{T}\right)^{a_{1} \ldots a_{h}}{ }_{b_{1} \ldots b_{n_{i}}}^{a_{h+1} \ldots a_{k}}=\tilde{T}^{a_{1} \ldots a_{h}}{ }_{b_{1} \ldots b_{n_{i}}}{ }^{a_{h+1} \ldots a_{k}}
$$

and

$$
\tilde{T}^{a_{1} \ldots a_{h}}{ }_{b_{1} \ldots b_{n_{i}}}^{a_{h+1} \ldots a_{k}}=-Y^{a_{1} \ldots a_{h}}{ }_{c b_{1} \ldots b_{n_{i}}}^{a_{h+1} \ldots a_{k} ; c}+(-1)^{n_{i}-1} n_{i} Z^{a_{1} \ldots a_{h}}{ }_{\left[b_{1} \ldots b_{n_{i}-1}{ }^{a_{h+1} \ldots a_{k}} ; b_{n_{i}}\right]}
$$

where $Y^{a_{1} \ldots a_{h}} b_{1} \ldots b_{n_{i}+1} a_{h+1} \ldots a_{k}$ is a rank- $(m+1)$ tensor of type $r$-fold $\left(n_{1}, \ldots, n_{i}+1, \ldots, n_{r}\right)$ form and $Z^{a_{1} \ldots a_{h}} b_{1} \ldots b_{n_{i}-1} a_{h+1} \ldots a_{k}$ is a rank- $(m-1)$ tensor of type $r$-fold $\left(n_{1}, \ldots, n_{i}-\right.$ $\left.1, \ldots, n_{r}\right)$-form, given respectively by

$$
\begin{aligned}
Y^{a_{1} \ldots a_{h}}{ }_{b_{1} \ldots b_{n_{i}+1}}^{a_{h+1} \ldots a_{k}} & \equiv(-1)^{n_{i}}\left(n_{i}+1\right) \stackrel{o}{T}^{a_{1} \ldots a_{h}}{ }_{\left[b_{1} \ldots b_{n_{i}}{ }^{a_{h+1} \ldots a_{k}}{ }_{\left.; b_{n_{i}+1}\right]}\right.}, \\
Z^{a_{1} \ldots a_{h}}{ }_{b_{1} \ldots b_{n_{i}-1}} & \equiv-T^{a_{1} \ldots a_{h}}{ }_{c b_{1} \ldots b_{n_{i}-1}}^{a_{h+1} \ldots a_{k} ; c}
\end{aligned}
$$

Trivially, these potentials in the above result are highly non-unique. For $r$-fold forms with $r>1$ we can repeat the above constructions for each $i \in\{1, \ldots, r\}$. Therefore the previous definition of potentials can be applied to each of the antisymmetric blocks, and thus there are many possible different pairs of potentials.

A second disadvantage is that each of the operators $\Delta_{(i)}, i=1,2, \ldots r$, in general, does not have all of the useful properties which we would like. Each operator is adapted to respect the index properties of its corresponding block, but not for the other blocks of indices. So, for instance, any index symmetry or trace property across the different blocks of a tensor will not necessarily be directly reflected in its superpotential.

\section{A weighted de Rham operator and associated po- tentials. Harmonic tensors.}

Of course, one can mix the operators $\Delta_{(i)}, i=1,2, \ldots r$, weighting them, and obtain new Laplace-type operators with similar or better properties. A particularly good one is,

Theorem 4.1 The operator $\bar{\Delta}$ given by

$$
\bar{\Delta} \equiv \frac{1}{r}\left(\Delta_{(1)}+\Delta_{(2)}+\ldots+\Delta_{(r)}\right)=\frac{1}{r} \sum_{i=1}^{r} \Delta_{(i)}
$$


is linear, self-adjoint with respect to the scalar product (14), respects all index symmetry properties, and commutes with all trace operations when acting on arbitrary tensor fields (here $r$ is the form-structure number of the tensor field). It is related to the Lichnerowicz operator by

$$
\bar{\Delta}=\frac{1}{r} \Delta_{L}-\frac{r-1}{r} \nabla^{c} \nabla_{c} .
$$

Proof. The linearity and self-adjointness follows from these properties for each of the $\Delta_{(i)}$. The formula (30) can be easily established from (13) and (22) (see also formulas (34) and (40,44) below). The commutativity with index permutations and traces then follows immediately from the properties of $\Delta_{L}$ and $\nabla^{c} \nabla_{c}$.

So we find that although $\Delta_{L}$ and $\bar{\Delta}$ do not coincide, they are closely related, and indeed $\bar{\Delta}$ has most of the useful properties of $\Delta_{L}$; but crucially $\bar{\Delta}$ in addition has direct links with $d_{(i)}$ and $\delta_{(i)}$. Hence we believe that $\bar{\Delta}$ is a more powerful alternative than the classical Lichnerowicz operator $\Delta_{L}$ since it is so well adapted to dealing with the $r$-fold form structure of the tensors. Of course, for single $p$-forms we have

$$
\bar{\Delta} \Sigma=\Delta \Sigma=\Delta_{L} \Sigma, \quad \forall \Sigma \in \Lambda^{p}
$$

An extremely important consequence of this new operator $\bar{\Delta}$ defined in (29) is that for

any given tensor field $T$, there exists an associated superpotential $\stackrel{\circ}{T}$, by which we mean a superpotential, not just with the same form-structure number and block ranks, but also with the same index symmetries and trace properties as T. Moreover, from this associated superpotential, a set of $2 r$ potentials can be obtained in a natural and straightforward manner from the definition of $\bar{\Delta}$

$$
\bar{\Delta} \stackrel{o}{T} \equiv \frac{1}{r} \sum_{i=1}^{r}\left[\delta_{(i)}\left(d_{(i)} \stackrel{o}{T}\right)+d_{(i)}\left(\delta_{(i)} \stackrel{o}{T}\right)\right] .
$$

Explicitly we have thus proven,

Theorem 4.2 Given any tensor field $T$, there always exists an associated superpotential $\stackrel{\circ}{T}$ such that $\bar{\Delta} \stackrel{o}{T}=T$; furthermore, if $r$ is the form-structure number of $T$, there always exists a set of $2 r$ local potentials $\left(Y_{(i)}, Z_{(i)}\right), i=1,2, \ldots r$, such that

$$
T=\frac{1}{r} \sum_{i=1}^{r}\left(\delta_{(i)} Y_{(i)}+d_{(i)} Z_{(i)}\right)
$$

where $Y_{(i)}=d_{(i)} \stackrel{o}{T}$ and $Z_{(i)}=\delta_{(i)} \stackrel{o}{T}$ are the potentials.

In our earlier derivation of the potential for the Weyl tensor [27] the operator which we constructed for the superpotential is the special case of $\bar{\Delta}$ for a double $(2,2)$-form. To the best of our knowledge, this seems to be the only time such a weighted de Rham operator $\bar{\Delta}$ has appeared in the literature. There are a variety of other generalised Laplacians [7, 52, 45], but for tensor-valued forms in pseudo-Riemannian spaces with Levi-Civita 
connections the only Laplacians mentioned are usually $-\nabla^{c} \nabla_{c}$, Lichnerowicz's Laplacian $\Delta_{L}$ and the Laplacians which we have labeled $\Delta_{(i)} .{ }^{2}$ In particular for double $(2,2)$-forms such as the Riemann tensor, the Laplacian used in [5, 44, 4, 9, 8, is the one we label $\Delta_{(2)}$, and as we argue in Section 7, this is not really a suitable operator in that context. It is only in [14] that any attempt is made to compare different Laplacians, where it is pointed out that, for the special case of a symmetric 2-tensor, the Lichnerowicz Laplacian can be written as $\Delta_{L}=\Delta_{(1)}+\Delta_{(2)}+\nabla^{c} \nabla_{c}$ (which can be considered as a special case of formula (30) ) ; but the significance of $\left(\Delta_{(1)}+\Delta_{(2)}\right)$ as a Laplacian was not recognised in [14.

We start with a simple logical definition which will allow us to show the potentialities of the new Laplacian $\bar{\Delta}$ with a very simple preliminary application.

Definition 4.1 A tensor field $T$ will be called harmonic if and only if

$$
\bar{\Delta} T=0
$$

Obviously, any fully harmonic tensor in the sense of Definition 3.2 is trivially harmonic. The converse, however, does not hold in general. Nevertheless, from formula (23) we deduce the general identity

$$
<T, \bar{\Delta} T>=\frac{1}{r} \sum_{i=1}^{r}<T, \Delta_{(i)} T>=\frac{1}{r} \sum_{i=1}^{r}\left(<d_{(i)} T, d_{(i)} T>+<\delta_{(i)} T, \delta_{(i)} T>\right)
$$

for arbitrary tensor fields. Therefore, it is straightforward to obtain the following converse in proper Riemannian manifolds.

Theorem 4.3 Let $\left(V_{n}, g\right)$ be a compact without boundary proper Riemannian manifold. Then, a tensor $T$ is harmonic if and only if it is fully harmonic, and if and only if

$$
d_{(i)} T=0, \quad \delta_{(i)} T=0, \quad \forall i \in\{1, \ldots, r\} .
$$

\section{Remarks}

- The above definition is different from the Lichnerowicz harmonic property which makes use of the condition $\Delta_{L} T=0$; and we emphasise that, in general, there is no analogue of this theorem by using $\Delta_{L}$.

- This definition differs from the harmonic definition in [39] for 2-fold forms in proper Riemannian space; in fact the definition there coincides with our definition of 1harmonic 2-fold forms, which is equivalent to $\Delta_{1} \omega=0$ where $\omega$ is a $(p, q)$-form.

- See Section 7 for details concerning the harmonic property of the Riemann tensor.

\footnotetext{
${ }^{2}$ To avoid any misunderstanding we point out that what we have called the 'usual Laplacian' $-\nabla^{c} \nabla_{c}$ (and with the symbol $\Delta_{(\ell)}$ in (21)) is sometimes given by the symbol $\bar{\Delta}$, 42, 52, and called the 'rough Laplacian' [52] or the 'naive Laplacian' 14].
} 
- The tensor decomposition into superpotentials and potentials for vectors and 2tensors developed in 32 exploited the Lichnerowicz operator $\Delta_{L}$ in order to find the superpotential $U$ for any tensor $V$ via $\Delta_{L} U=V$; however, such a superpotential $U$ does not, in general, yield potentials in a straightforward manner as there is no direct natural link to any first order operators.

For general calculations, we would need the commutation properties of the operators $d_{(i)}$ and $\delta_{(j)}$. Actually the general case requires a detailed and cumbersome study in nonflat backgrounds; but we are only interested in the case of double forms $(r=2)$ in this paper, and so we devote the next section to this case in full detail.

\section{$5 \quad$ Double $(q, p)$-forms}

In this section we deal with tensors with form-structure number $r=2$ and block ranks $n_{1}=q$ and $n_{2}=p$, called double $(q, p)$-forms. These are tensors with $p+q$ indices which fall into two categories: a block of $q$ antisymmetric indices, and another block of $p$ antisymmetric indices. By rearranging if necessary, the tilded version of the tensor can then always be written as

$$
\tilde{T}^{a_{1} \ldots a_{q}} b_{1} \ldots b_{p}=\tilde{T}^{\left[a_{1} \ldots a_{q}\right]_{\left[b_{1} \ldots b_{p}\right]}} .
$$

In addition to the Lichnerowicz operator, there are three other Laplace-like operators $\Delta_{(1)}, \Delta_{(2)}$ and $\bar{\Delta}$ as defined in the previous sections acting on these objects. Using (18), (22), and (29) their explicit formulas are

$$
\begin{aligned}
& \left(\Delta_{(1)} T\right)^{a_{1} \ldots a_{q}} b_{1} \ldots b_{p}=-\nabla^{c} \nabla_{c} \tilde{T}_{a_{1} \ldots a_{q} \ldots b_{p}}^{a_{1}}+q R^{c\left[a_{1}\right.} \tilde{T}_{c}^{\left.a_{2} \ldots a_{q}\right]_{b_{1} \ldots b_{p}}} \\
& -\frac{q(q-1)}{2} R^{c d\left[a_{1} a_{2}\right.} \tilde{T}_{c d}{ }^{\left.a_{3} \ldots a_{q}\right]}{ }_{b_{1} \ldots b_{p}}+q p R^{\left[a_{1}\right.}{ }_{c d\left[b_{1}\right.} \tilde{T}_{\left.b_{2} \ldots a_{p}\right]}^{\left.a_{2} \ldots a_{q}\right] c d} \\
& \left(\Delta_{(2)} T\right)^{a_{1} \ldots a_{q}}{ }_{b_{1} \ldots b_{p}}=-\nabla^{c} \nabla_{c} \tilde{T}_{b_{1} \ldots a_{p}}^{a_{1} \ldots b_{p}}+p R_{c\left[b_{1}\right.} \tilde{T}_{\left.b_{2} \ldots b_{p}\right]}^{a_{1} \ldots a_{q} c} \\
& -\frac{p(p-1)}{2} R_{c d\left[b_{1} b_{2}\right.} \tilde{T}_{\left.b_{3} \ldots b_{p}\right]}^{a_{1} \ldots a_{q} c d}+p q R^{\left[a_{1}\right.}{ }_{c d\left[b_{1}\right.} \tilde{T}_{\left.b_{2} \ldots b_{p}\right]}^{\left.a_{2} \ldots a_{q}\right] c d} \text {; } \\
& (\bar{\Delta} T)_{b_{1} \ldots b_{p}}^{a_{1} \ldots a_{q}}=-\nabla^{c} \nabla_{c} \tilde{T}_{b_{1} \ldots b_{p}}^{a_{1} \ldots a_{q}}+\frac{q}{2} R^{c\left[a_{1}\right.} \tilde{T}_{c}^{\left.a_{2} \ldots a_{q}\right]}{ }_{b_{1} \ldots b_{p}}+\frac{p}{2} R_{c\left[b_{1}\right.} \tilde{T}_{\left.b_{2} \ldots b_{p}\right]}^{a_{1} \ldots a_{q} c} \\
& -\frac{q(q-1)}{4} R^{c d\left[a_{1} a_{2}\right.} \tilde{T}_{c d}^{\left.a_{3} \ldots a_{q}\right]_{b_{1} \ldots b_{p}}}-\frac{p(p-1)}{4} R_{c d\left[b_{1} b_{2}\right.} \tilde{T}_{\left.{ }_{3} \ldots b_{p}\right]}^{a_{1} \ldots a_{q} c d} \\
& +q p R^{\left[a_{1}\right.}{ }_{c d\left[b_{1}\right.} \tilde{T}^{\left.a_{2} \ldots a_{q}\right] c d}{ }_{\left.b_{2} \ldots b_{p}\right]}
\end{aligned}
$$

The same comments as before for the cases with $p$ or $q$ equal to $1, n-1$ or $n$ are in order

here. We also note from formula (30) in Theorem 4.1 that $\bar{\Delta}$ is related to the Lichnerowicz operator in this case by

$$
\bar{\Delta}=\frac{1}{2}\left(\Delta_{L}-\nabla_{c} \nabla^{c}\right)
$$

Specialising Theorem 4.2 we obtain, 
Corollary 5.1 Given any tensor field $T$ with the structure of a double $(q, p)$-form there always exists an associated local superpotential $\stackrel{\circ}{T}$ such that $\bar{\Delta} \stackrel{o}{T}=T$; furthermore there always exist local potentials $Y_{(1)}, Y_{(2)}, Z_{(1)}, Z_{(2)}$ such that

$$
T=\frac{1}{2}\left(\delta_{(1)} Y_{(1)}+\delta_{(2)} Y_{(2)}+d_{(1)} Z_{(1)}+d_{(2)} Z_{(2)}\right)
$$

where the double $(q+1, p)$-form $Y_{(1)}=d_{(1)} \stackrel{o}{T}$, the double $(q, p+1)$-form $Y_{(2)}=d_{(2)} \stackrel{o}{T}$, the double $(q-1, p)$-form $Z_{(1)}=\delta_{(1)} \stackrel{o}{T}$ and the double $(q, p-1)$-form $Z_{(2)}=\delta_{(2)} \stackrel{o}{T}$.

In the sequel we will need to deal with contractions as well as permutations of indices for different expressions, so we make two formal definitions which will be useful.

Definition 5.1 (Trace of a double form) The trace of a double $(q, p)$-form $T$ is the double $(q-1, p-1)$-form $\operatorname{tr}(T)$ given by

$$
(\widetilde{\operatorname{tr}(T)}){ }^{a_{2} \ldots a_{q}} b_{2} \ldots b_{p} \equiv \tilde{T}^{c a_{2} \ldots a_{q}} c_{2} \ldots b_{p} .
$$

Once more, we must remark that if $q=1$ ( or $p=1$ ), then the first (second) block disappears after taking the trace tr, so that the resulting tensor has a form-structure number less than 2. In these situations, and as happened in other situations explained before, sometimes it is necessary to consider the resulting tensor $\operatorname{tr}(T)$ as an equivalent double $(0, p-1)$-form (or double $(q-1,0)$-form). Bearing this in mind, some useful results follow immediately from the respective definitions (16) and (201),

$$
\begin{array}{ll}
\operatorname{tr}\left(d_{(1)} T\right)=-d_{(1)} \operatorname{tr}(T)-\delta_{(2)} T, & \operatorname{tr}\left(d_{(2)} T\right)=-d_{(2)} \operatorname{tr}(T)-\delta_{(1)} T, \\
\operatorname{tr}\left(\delta_{(1)} T\right)=-\delta_{(1)} \operatorname{tr}(T)(q \geq 2) ; & \operatorname{tr}\left(\delta_{(2)} T\right)=-\delta_{(2)} \operatorname{tr}(T) \quad(p \geq 2) .
\end{array}
$$

In [39] the second of equations (37) was used as the definition of $\delta_{(1)}$ for a double $(p, q)$ form.

Definition 5.2 (Transpose of a double form) For a double (q,p)-form $T$ we define the (generalised) transpose ${ }^{t} T$ of $T$ as the double $(p, q)$-form given by interchange of the blocks:

$$
\left(\widetilde{{ }^{t} T}\right)^{a_{1} \ldots a_{p}}{ }_{b_{1} \ldots b_{q}} \equiv \tilde{T}_{b_{1} \ldots b_{q}}{ }^{a_{1} \ldots a_{p}} .
$$

Obviously ${ }^{t t} T=T$, and the following useful results also follow from the definitions

$$
d_{(2)}\left({ }^{t} T\right)={ }^{t}\left(d_{(1)} T\right), \quad \delta_{(2)}\left({ }^{t} T\right)={ }^{t}\left(\delta_{(1)} T\right)
$$

In addition, we note that the operations of transpose and trace commute,

$$
{ }^{t}(\operatorname{tr}(T))=\operatorname{tr}\left({ }^{t} T\right)
$$

Of course, we could also define traces and transposes of the more general $r$-fold forms in the previous section, by taking pairs of blocks at a time. 
For future reference we give explicitly the five cases of $(1,1)-,(2,1)-,(2,2)-,(2,3)$ and $(2,4)$-forms respectively for (34)

$$
\begin{aligned}
& -(\bar{\Delta} T)^{a_{1}}{ }_{b_{1}}=\nabla^{c} \nabla_{c} T_{{ }_{b_{1}}}^{a_{1}}-\frac{1}{2} R^{c a_{1}} T_{c b_{1}}-\frac{1}{2} R_{c b_{1}} T^{a_{1} c}-R^{a_{1}}{ }_{c d b_{1}} T^{c d} \\
& -(\bar{\Delta} T)_{b_{1}}^{a_{1} a_{2}}=\nabla^{c} \nabla_{c} \tilde{T}_{b_{1}}^{a_{1} a_{2}}-R^{c\left[a_{1}\right.} \tilde{T}_{c}^{\left.a_{2}\right]} b_{1}+\frac{1}{2} R^{c d a_{1} a_{2}} \tilde{T}_{c d b_{1}} \\
& -\frac{1}{2} R_{c b_{1}} \tilde{T}^{a_{1} a_{2} c}-2 R^{\left[a_{1}\right.}{ }_{c d b_{1}} \tilde{T}^{\left.a_{2}\right] c d} \\
& -(\bar{\Delta} T)^{a_{1} a_{2}} b_{1} b_{2}=\nabla^{c} \nabla_{c} \tilde{T}_{b_{1} b_{2}}^{a_{1} a_{2}}-R^{c\left[a_{1}\right.} \tilde{T}_{c}^{\left.a_{2}\right]}{ }_{b_{1} b_{2}}+\frac{1}{2} R^{c d a_{1} a_{2}} \tilde{T}_{c d b_{1} b_{2}} \\
& -R_{c\left[b_{1}\right.} \tilde{T}_{\left.b_{2}\right]}^{a_{1} a_{2} c}+\frac{1}{2} R_{c d b_{1} b_{2}} \tilde{T}^{a_{1} a_{2} c d}-4 R_{c d\left[b_{1}\right.}^{\left[a_{1}\right.} \tilde{T}^{\left.a_{2}\right] c d} b_{\left.b_{2}\right]} \\
& -(\bar{\Delta} T)_{b_{1} b_{2} b_{3}}^{a_{1} a_{2}}=\nabla^{c} \nabla_{c} \tilde{T}_{b_{1} b_{2} b_{3}}^{a_{1} a_{2}}-R^{c\left[a_{1}\right.} \tilde{T}_{c}^{a_{2} b_{1} b_{2} b_{3}}+\frac{1}{2} R^{c d a_{1} a_{2}} \tilde{T}_{c d b_{1} b_{2} b_{3}} \\
& -\frac{3}{2} R_{c\left[b_{1}\right.} \tilde{T}_{\left.b_{2} b_{3}\right]}^{a_{1} a_{2} c}+\frac{3}{2} R_{c d\left[b_{1} b_{2}\right.} \tilde{T}_{\left.b_{3}\right]}^{a_{1} a_{2} c d}-6 R^{\left[a_{1}\right.}{ }_{c d\left[b_{1}\right.} \tilde{T}_{\left.b_{2} b_{3}\right]}^{\left.a_{2}\right] c} \\
& -(\bar{\Delta} T)_{b_{1} b_{2} b_{3} b_{4}}^{a_{1} a_{2}}=\nabla^{c} \nabla_{c} \tilde{T}^{a_{1} a_{2}} b_{1} b_{2} b_{3} b_{4}-R^{c\left[a_{1}\right.} \tilde{T}_{c}^{\left.a_{2}\right]} b_{1} b_{2} b_{3} b_{4}+\frac{1}{2} R^{c d a_{1} a_{2}} \tilde{T}_{c d b_{1} b_{2} b_{3} b_{4}} \\
& -2 R_{c\left[b_{1}\right.} \tilde{T}_{\left.b_{2} b_{3} b_{4}\right]}^{a_{1} a_{2} c}+3 R_{c d\left[b_{1} b_{2}\right.} \tilde{T}_{\left.b_{3} b_{4}\right]}^{a_{1} a_{2} c d}{ }_{c d\left[b_{1}\right.} \tilde{T}_{\left.b_{2} b_{3} b_{4}\right]}^{\left.a_{2}\right] c d}
\end{aligned}
$$

For the case of double $(q, p)$-forms, a straightforward computation provides the commutation properties of the operators $d_{(i)}$ and $\delta_{(j)}$ for $i, j \in\{1,2\}$

$$
\begin{gathered}
\left(\left[d_{(1)}, d_{(2)}\right] T\right)^{a_{1} \ldots a_{q+1}}{ }_{b_{1} \ldots b_{p+1}}=\frac{(-1)^{p+q}}{2}(p+1)(q+1) \times \\
\left(q R_{c\left[b_{p+1}\right.}^{\left[a_{q} a_{q+1}\right.} \tilde{T}^{\left.a_{1} \ldots a_{q-1}\right] c}{ }_{\left.b_{1} \ldots b_{p}\right]}-p R^{c\left[a_{q+1}\right.}\left[b_{p} b_{p+1} \tilde{T}^{\left.a_{1} \ldots a_{q}\right]}{ }_{\left.b_{1} \ldots b_{p-1}\right] c}\right),\right. \\
\left(\left[d_{(1)}, \delta_{(2)}\right] T\right)^{a_{1} \ldots a_{q+1}}{ }_{b_{1} \ldots b_{p-1}}=(-1)^{q}(q+1)\left(\frac{q}{2} R_{d}^{c}{ }_{d}^{\left[a_{q} a_{q+1}\right.} \tilde{T}^{\left.a_{1} \ldots a_{q-1}\right] d}{ }_{c b_{1} \ldots b_{p-1}}\right. \\
\left.+\frac{p-1}{2} R_{\left[b_{1}{ }^{\left[a_{q+1}\right.} \tilde{T}^{\left.a_{1} \ldots a_{q}\right]} b_{2} \ldots b_{p-1}\right] c d}+R^{d\left[a_{q+1}\right.} \tilde{T}^{\left.a_{1} \ldots a_{q}\right]}{ }_{d b_{1} \ldots b_{p-1}}\right), \\
\left(\left[\delta_{(1)}, \delta_{(2)}\right] T\right)^{a_{1} \ldots a_{q-1}}{ }_{b_{1} \ldots b_{p-1}}=\frac{p-1}{2} R_{d\left[b_{1}\right.}^{c e} \tilde{T}^{d a_{1} \ldots a_{q-1}}{ }_{\left.b_{2} \ldots b_{p-1}\right] c e} \\
-\frac{q-1}{2} R_{c e}^{d\left[a_{1}\right.} \tilde{T}^{\left.a_{2} \ldots a_{q-1}\right] c e}{ }_{d b_{1} \ldots b_{p-1}}
\end{gathered}
$$

(Observe again, that in flat space these operators commute.) In will be useful in subsequent calculations to note from (33) and (34)

$$
\begin{array}{r}
\left.\left(\Delta_{(1)} T\right)^{a_{1} \ldots a_{q}} b_{1} \ldots b_{p}-\left(\Delta_{(2)} T\right)^{a_{1} \ldots a_{q}} b_{1} \ldots b_{p}=q R^{c\left[a_{1}\right.} \tilde{T}_{c}{ }^{\left.a a_{2} \ldots a_{q}\right]} b_{b_{1} \ldots b_{p}}-p R_{c\left[b_{1}\right.} \tilde{T}^{a_{1} \ldots a_{q} c} b_{2} \ldots b_{p}\right] \\
\left.-\frac{q(q-1)}{2} R^{c d\left[a_{1} a_{2}\right.} \tilde{T}_{c d}{ }^{\left.a_{3} \ldots a_{q}\right]} b_{1} \ldots b_{p}+\frac{p(p-1)}{2} R_{c d\left[b_{1} b_{2}\right.} \tilde{T}^{a_{1} \ldots a_{q} c d} b_{3} \ldots b_{p}\right] .
\end{array}
$$




\section{Double $(p, p)$-forms: Curvature tensors}

The transpose ${ }^{t} T$ of $T$ is of special relevance for the special case of the double $(q, p)$-forms in which both blocks have the same number of indices, say $p$ - these double $(p, p)$-forms include, of course, the important case of curvature tensors. In this case the two blocks of $T$ can be interchanged, and hence we will say that a double $(p, p)$-form is symmetric if $T={ }^{t} T$, and antisymmetric if $T=-{ }^{t} T$ (in this case only for $p>1$ ). Of course, for $p>1$ any double $(p, p)$-form can be decomposed uniquely into a symmetric and an antisymmetric one (if $p=1$ the decomposition gives a 2 -form and a double symmetric $(1,1)$-form). Hence, without loss of generality, in what follows we will only consider these two cases,

$$
T= \pm{ }^{t} T \quad \text { or } \quad \tilde{T}^{a_{1} \ldots a_{p}}{ }_{b_{1} \ldots b_{p}}= \pm \tilde{T}_{b_{1} \ldots b_{p}}^{a_{1} \ldots a_{p}} .
$$

Then it follows trivially from (39) that

$$
d_{(2)} T= \pm{ }^{t}\left(d_{(1)} T\right), \quad \delta_{(2)} T= \pm{ }^{t}\left(\delta_{(1)} T\right)
$$

and therefore $\Delta_{(2)} T= \pm^{t}\left(\Delta_{(1)} T\right)$ so that

$$
\bar{\Delta} T=\frac{1}{2}\left(\Delta_{(2)} T \pm^{t}\left(\Delta_{(2)} T\right)\right) .
$$

Since the associated superpotential $\stackrel{\circ}{T}$ will have the same symmetry properties as $T$, it follows that the four potentials $Y_{(1)}, Y_{(2)}, Z_{(1)}$ and $Z_{(2)}$ defined in the previous section satisfy

$$
\begin{aligned}
& Y_{(2)}= \pm^{t} Y_{(1)} \equiv(-1)^{p+1} Y, \\
& Z_{(2)}= \pm^{t} Z_{(1)} \equiv(-1)^{p-1} Z,
\end{aligned}
$$

where furthermore the completely antisymmetric part $\mathcal{A}\left[Y_{(2)}\right]$ of $Y_{(2)}$ (or $Y$ ) may vanish identically due to the (anti)symmetry of the associated superpotential:

$$
\mathcal{A}\left[Y_{(2)}\right]=0 \text { for } \begin{cases}T={ }^{t} T & p \text { odd } \\ T=-{ }^{t} T & p \text { even. }\end{cases}
$$

The index notation for this restriction is simply

$$
Y_{\left[a_{1} \ldots a_{p} b_{1} \ldots b_{p+1}\right]}=0 \text { for } \begin{cases}T={ }^{t} T & p \text { odd } \\ T=-{ }^{t} T & p \text { even }\end{cases}
$$

Theorem 6.1 Given any tensor $T$ with the structure of a double (anti)symmetric $(p, p)$ form there always exists an associated local superpotential $\stackrel{\circ}{T}$ such that $\bar{\Delta} \stackrel{\circ}{T}=T$; furthermore there always exist a pair of local potentials $Y_{(2)}, Z_{(2)}$ satisfying (52) such that

$$
T=\frac{1}{2}\left[\delta_{(2)} Y_{(2)} \pm{ }^{t}\left(\delta_{(2)} Y_{(2)}\right)+d_{(2)} Z_{(2)} \pm{ }^{t}\left(d_{(2)} Z_{(2)}\right)\right],
$$


where the double $(p, p+1)$-form $Y_{(2)}=d_{(2)} \stackrel{o}{T}$, and the double $(p, p-1)$-form $Z_{(2)}=\delta_{(2)} \stackrel{o}{T}$.

Using the notation introduced in (50) and (51), (54) can be written in index notation as

$$
\begin{aligned}
\tilde{T}_{b_{1} \ldots b_{p}}^{a_{1} \ldots a_{p}}= & \frac{1}{2}\left(Y_{b_{1} \ldots b_{p} c}^{a_{1} \ldots a_{p}} \pm Y_{b_{1} \ldots b_{p}{ }^{a_{1} \ldots a_{p} c}{ }_{; c}}\right. \\
& \left.+p Z^{a_{1} \ldots a_{p}}{ }_{\left[b_{1} \ldots b_{p-1} ; b_{p}\right]} \pm p Z_{b_{1} \ldots b_{p}}\left[a_{1} \ldots a_{p-1} ; a_{p}\right]\right)
\end{aligned}
$$

where the potential $Y$ satisfies (53), and the potentials themselves can be given in terms of the double (anti)symmetric associated superpotential ${ }^{\circ}$ by

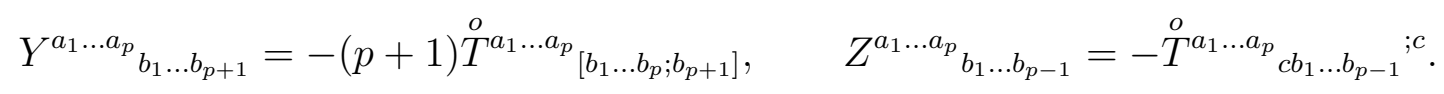

Since the operations of transpose and trace commute, then if $T$ is (anti)symmetric, so is $\operatorname{tr}(T)$. Suppose now that, in addition to the (anti)symmetry between blocks, the double $(p, p)$-form $T$ is traceless, i.e.,

$$
\operatorname{tr}(T)=0
$$

The associated superpotential $\stackrel{o}{T}$ will also be traceless with

$$
\operatorname{tr}(\stackrel{o}{T})=0
$$

But then the potentials $Y_{(2)}$ and $Z_{(2)}$ of (54) are not independent and one can easily check using (57) that $Z_{(2)}$ is essentially the trace of the transpose of $Y_{(2)}$ :

$$
Z_{(2)}=\mp \operatorname{tr}\left({ }^{t} Y_{(2)}\right)
$$

or with indices and the notation of (50,51)

$$
Z_{b_{1} \ldots b_{p-1}}^{a_{1} \ldots a_{p}}=\mp Y_{c b_{1} \ldots b_{p-1}} c a_{1} \ldots a_{p}
$$

Furthermore

$$
\operatorname{tr}\left(\operatorname{tr}\left(Y_{(2)}\right)\right)=0
$$

which becomes in index notation

$$
Y_{c d b_{1} \ldots b_{p-2}} c d a_{1} \ldots a_{p-1}=0
$$

Therefore, we have

Theorem 6.2 Given any tensor $T$ with the structure of a double (anti)symmetric traceless $(p, p)$-form there always exists an associated local superpotential $\stackrel{\circ}{T}$ such that $\bar{\Delta} \stackrel{o}{T}=T$; furthermore, there always exists a double $(p, p+1)$-form local potential $Y_{(2)}$ satisfying (52) and $\operatorname{tr}\left(\operatorname{tr}\left(Y_{(2)}\right)\right)=0$ such that

$$
T=\frac{1}{2}\left(\delta_{(2)} Y_{(2)} \pm{ }^{t}\left(\delta_{(2)} Y_{(2)}\right)-d_{(1)} \operatorname{tr}\left(Y_{(2)}\right) \mp{ }^{t}\left(d_{(1)} \operatorname{tr}\left(Y_{(2)}\right)\right)\right),
$$


where the double $(p, p+1)$-form $Y_{(2)}=d_{(2)} \stackrel{o}{T}$.

With the notation introduced in (50), the index version of (60) can be written as

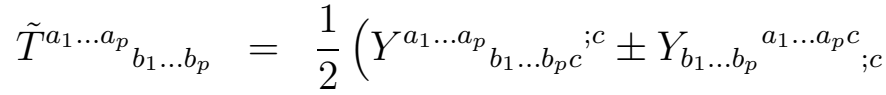

$$
\begin{aligned}
& \left.\left.-p Y^{c\left[a_{1} \ldots a_{p-1}\right.} c b_{1} \ldots b_{p}{ }^{\left.; a_{p}\right]} \mp p Y_{c\left[b_{1} \ldots b_{p-1}\right.}{ }^{c a_{1} \ldots a_{p}} ; b_{p}\right]\right)
\end{aligned}
$$

where the potential $Y$ satisfies (53) and (59), and is given in terms of the double (anti)symmetric traceless associated superpotential $\stackrel{\circ}{T}$ by

$$
Y^{a_{1} \ldots a_{p}}{ }_{b_{1} \ldots b_{p+1}} \equiv-(p+1) \stackrel{o}{T^{a_{1} \ldots a_{p}}}\left[b_{1} \ldots b_{p} ; b_{p+1}\right] .
$$

In 23 it was shown in $2 m$ dimensions that a traceless symmetric $(m, m)$-form has a traceless $(m, m-1)$-form potential. That result is a special case of the much more general result in this theorem, where the double dual of the $(m, m-1)$-form potential in [23] is precisely the $(m, m+1)$-form potential $Y$ in this theorem.

For future reference we give explicitly the index version of the potential structure for a symmetric 2 -tensor

$$
T_{a b}=\frac{1}{2}\left(Y_{a b c}{ }^{; c}+Y_{b a c}{ }^{; c}+Z_{a ; b}+Z_{b ; a}\right) .
$$

and for a traceless symmetric 2-tensor

$$
T_{a b}=\frac{1}{2}\left(Y_{a b c}{ }^{; c}+Y_{b a c}{ }^{; c}-Y_{c a ; b}^{c}-Y_{c b ; a}^{c}\right) .
$$

where (in both cases) the double $(1,2)$-form $Y_{b c}^{a}$ satisfies $Y_{[a b c]}=0$.

There is a well known decomposition for symmetric 2-tensors in three dimensional spaces, but it is restricred to proper Riemannian space [17, 54, and so differs from this one. Of course, the antisymmetric 2-tensor is just a single 2-form whose decomposition (8) is easily seen to agree with the decomposition which follows from Theorem 6.1.

\subsection{Application to general curvature tensors}

Let us apply the above results to the case of Riemann candidates, that is, tensors with the algebraic properties of a Riemann curvature tensor. Let $\mathcal{R}_{a b c d}$ be any such Riemann candidate, that is to say

$$
\mathcal{R}_{a b c d}=\mathcal{R}_{[a b][c d]}, \quad \mathcal{R}_{a[b c d]}=0\left(\Longrightarrow \mathcal{R}_{a b c d}=\mathcal{R}_{c d a b}\right)
$$

so that $\mathcal{R}_{a b c d}$ is in particular a symmetric double $(2,2)$-form.

Let $\stackrel{o}{\mathcal{R}}_{a b c d}$ be the associated local superpotential for $\mathcal{R}_{a b c d}$ by

$$
(\bar{\Delta} \stackrel{o}{\mathcal{R}})_{a b c d}=\mathcal{R}_{a b c d}
$$


and because of the properties of $\bar{\Delta}, \stackrel{o}{\mathcal{R}}$ is also a Riemann candidate; the pair of potentials defined in Theorem 6.1 become now

$$
Y_{a b c d e}=Y_{[a b][c d e]} \equiv-3 \stackrel{o}{\mathcal{R}}_{a b[c d ; e]}, \quad Z_{a b c}=Z_{[a b] c} \equiv-\stackrel{o}{\mathcal{R}}_{a b d c} ; d
$$

It is important to realize, since $\stackrel{\circ}{\mathcal{R}}$ is a Riemann candidate satisfying (65), that these potentials satisfy the additional symmetries

$$
Y_{a[b c d e]}=0, \quad Z_{[a b c]}=0,
$$

the first of which implies the following useful properties

$$
Y_{[b c d] e}^{e}=0, \quad Y_{[a b c d] e}=0, \quad Y_{a b c d e}=3 Y_{[c d e] a b}=3 Y_{a[c d e] b}, \quad Y_{a[b c] d e}=-Y_{a[d e] b c}
$$

Given that (66) always has local solutions $\stackrel{\circ}{\mathcal{R}}$ for any given $\mathcal{R}$ we have,

Theorem 6.3 Any Riemann candidate tensor $\mathcal{R}^{a b}{ }_{c d}$ has a pair of local potentials given by a double $(2,3)$-form $Y^{a b}{ }_{\text {cde }}$ and a double (2,1)-form $Z^{a b}{ }_{c}$ with the properties (68) such that

$$
\mathcal{R}_{a b c d}=\frac{1}{2}\left(Y_{a b c d e}{ }^{i e}+Y_{c d a b e^{; e}}+2 Z_{a b[c ; d]}+2 Z_{c d[a ; b]}\right) .
$$

The potentials themselves can be given in terms of the associated (Riemann candidate) local superpotential $\stackrel{\circ}{\mathcal{R}}_{a b c d}$ by (67).

Of course, any Riemann candidate can be decomposed in terms of its trace (its 'Ricci tensor') and its traceless part (its 'Weyl tensor'). The trace is a double symmetric $(1,1)$ form, and therefore the general Theorem 6.1 applies, and in particular (63). After a little rearranging, it can be seen that the potentials for this Ricci part are essentially the traces of the pair of potentials defined in (67) and the formula relating them is the trace of (701).

Corollary 6.1 Any Ricci candidate tensor $\mathcal{R}_{a b}$ has a pair of local potentials given by a double $(1,2)$-form $Y_{a b}^{c}$ with the property $Y_{[a b c]}=0$ and a double (1,0)-form $Z_{a}$ such that

$$
\mathcal{R}_{a b}=\frac{1}{2}\left(Y_{a b e} e^{i e}+Y_{b a e} e^{i e}+Z_{a ; b}+Z_{b ; a}\right)
$$

The relation with the Riemann candidate local potentials of (70) is

$$
Y_{a b}^{c}=Y_{e a b}^{e c}-Z_{a b}^{c}, \quad Z_{a}=Z_{e a}^{e} .
$$

The traceless part of the Ricci candidate tensor (essentially any traceless symmetric 2tensor) has an even simpler structure requiring only one potential, as shown in (64): 
Corollary 6.2 Any traceless Ricci candidate tensor $\hat{\mathcal{R}}_{a b}=\mathcal{R}_{a b}-\frac{1}{n} g_{a b} \mathcal{R}$, where $\mathcal{R}=\mathcal{R}_{c}^{c}$, has a local potential given by a double $(1,2)$-form $\hat{Y}_{a b}^{c}$ with the property $\hat{Y}_{[a b c]}=0$ such that

$$
\hat{\mathcal{R}}_{a b}=\frac{1}{2}\left(\hat{Y}_{a b e}{ }^{; e}+\hat{Y}_{b a e^{; e}}-\hat{Y}_{c a ; b}^{c}-\hat{Y}_{c b ; a}^{c}\right) .
$$

The relation with the Ricci candidate local potentials of (71) is

$$
\hat{Y}_{a b}^{c}=Y_{a b}^{c}+\frac{2}{n} g_{c[a} Y_{b] e}^{e}+\frac{2}{n} g_{c[a} Z_{b]} .
$$

More interesting is the traceless part of a Riemann candidate, which we call a Weyl candidate, that is, a double $(2,2)$-form $\mathcal{C}_{a b c d}=\mathcal{C}_{[a b] c d}=\mathcal{C}_{a b[c d]}$ with the algebraic properties of the Weyl conformal curvature tensor:

$$
\mathcal{C}^{a}{ }_{b c a}=0, \quad \mathcal{C}_{a[b c d]}=0, \quad\left(\Longrightarrow \mathcal{C}_{a b c d}=\mathcal{C}_{c d a b}\right)
$$

so that $\mathcal{C}_{a b c d}$ is in particular a traceless and symmetric double (2,2)-form. By considering

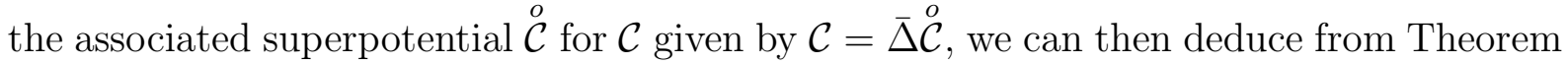
6.2 and Theorem 6.3 .

Theorem 6.4 Any Weyl candidate tensor field $\mathcal{C}_{\text {abcd }}$ has a double (2,3)-form local potential $P_{\text {abcde }}$ with the properties

$$
P_{a[b c d e]}=0, \quad P_{a b c}^{a b}=0
$$

such that

$$
\left.\mathcal{C}^{a b}{ }_{c d}=\frac{1}{2}\left(P^{a b}{ }_{c d e}{ }^{i e}+P_{c d}{ }^{a b e} ; e-2 P_{e[c}{ }^{a b e} ; d\right]-2 P^{e[a}{ }_{c d e}^{; b]}\right) .
$$

The potential itself can be given in terms of an associated local (Weyl candidate) superpotential $\stackrel{o}{\mathcal{C}}^{a b}{ }_{c d}$ by

$$
P^{a b}{ }_{c d e}=P^{[a b]}{ }_{[c d e]} \equiv-3 \stackrel{o}{\mathcal{C}}^{a b}{ }_{[c d ; e]} .
$$

This result was originally obtained, by a more direct route, in [27]. The operator $\bar{\Delta}$ which we have used in this theorem, specialising via (42), is easily seen to coincide with the operator which we constructed for the superpotential of the Weyl candidate tensor in [27. As noted there, in four dimensions our new $(2,3)$-form potential $P^{a b}{ }_{c d e}$ coincides with the double dual of the classical Lanczos $(2,1)$-form potential $H^{a b}{ }_{c}$ for the Weyl tensor [40].

Immediate consequences from the first of (174) are the following useful properties, reminiscent of (69)

$$
P_{[b c d] e}^{e}=0, \quad P_{[a b c d] e}=0, \quad P_{c d e}^{a b}=3 P_{[c d e]}^{a b}=3 P_{[c d e]}^{[a]}, \quad P_{a[b c] d e}=-P_{a[d e] b c} .
$$

The basic equation (75) can be written in several alternative but equivalent forms by using properties (74) and (77). Other possibilities are

$$
\left.\left.\mathcal{C}^{a b}{ }_{c d}=P^{a b}{ }_{c d e} e^{; e}+P^{e[a b]}{ }_{c d ; e}-P_{e[c}{ }^{a b e} ; d\right]-P^{e[a}{ }_{c d e} ; b\right]
$$

and

$$
\mathcal{C}^{a b}{ }_{c d}=P^{a b}{ }_{c d e} e^{; e}+P^{e[a b]}{ }_{c d ; e}-P^{e[a}{ }_{c d e^{; b]}}-2 P^{e[a b]}{ }_{e[c ; d]} .
$$




\section{Laplace-type equation for the Riemann tensor}

The intrinsic, as well as quickest and simplest, way to obtain a Laplace-like equation (a wave equation in Lorentzian signature) for the electromagnetic field $F$ is as follows. The 2 -form $F$ satisfies the Maxwell equations

$$
d F=0, \quad \delta F=J
$$

where $J$ is the electric current 1-form. By using the de Rham operator the Laplace-like equation follows from these equations strightforwardly

$$
\Delta F=d J .
$$

As is known, in the absence of electric charges and currents $(J=0)$ one has the simpler equation

$$
J=0 \Longrightarrow \Delta F=0 .
$$

The index version of (81) is the well-known equation ("wave" equation in Lorentzian signature)

$$
\nabla^{c} \nabla_{c} F^{a b}+R^{e f a b} F_{e f}-R^{a e} F_{e}^{b}+R^{b e} F_{e}^{a}=J^{[a ; b]}
$$

or equivalently

$$
\nabla^{c} \nabla_{c} F^{a b}-2 R^{e a f b} F_{e f}-R^{a e} F_{e}^{b}+R^{b e} F_{e}^{a}=J^{[a ; b]} .
$$

As has been always the case, the electromagnetic field may serve as source of inspiration for the gravitational field, and thereby for the curvature tensor of any pseudo-Riemannian manifold. Thus, there have been some attempts to formulate the analogue of (81) for the Riemann tensor with a generalised de Rham operator, using the Bianchi identities as the analogue to the Maxwell equations (801). We will use $\Re$ to represent the double symmetric $(2,2)$-form defined by the Riemann tensor $R_{c d}^{a b}$. Then, the Bianchi identities $R_{a b[c d ; e]}=0$ can be written in a concise form using the notation of this paper as

$$
d_{(2)} \Re=0 .
$$

Hence, using (37) to take the trace of (82) we readily obtain

$$
\delta_{(1)} \Re=-d_{(2)} \Re i c\left(=d_{(2)} \operatorname{tr}(\Re)\right)
$$

where $\Re i c=\operatorname{tr}(\Re)$ denotes the double symmetric $(1,1)$-form representing the Ricci tensor $R_{a b}$. (Alternatively (83) could be obtained by translating from the contracted index version of the Bianchi identities $R_{a b c d^{; a}}=-2 R_{b[c ; d]}$.)

The symmetry of the Riemann tensor ${ }^{t} \Re=\Re$ implies that (82) is actually equivalent, via (49), to

$$
d_{(1)} \Re=0
$$

and by taking the trace we obtain

$$
\delta_{(2)} \Re=-d_{(1)} \Re i c
$$


(or equivalently using the symmetry of the Riemann tensor in (83)).

By following a procedure similar to that leading to (80) exploiting the de Rham operator for tensor valued 2-forms [8, 9, 44, 4, which in our notation is simply $\Delta_{(2)}\left(\right.$ or $\left.\Delta_{(1)}\right)$, by using (82 85), one easily derives

$$
\Delta_{(2)} \Re=-d_{(2)} d_{(1)} \Re i c .
$$

For those spaces with $\nabla_{[a} R_{b] c}=0$,

$$
d_{(1)} \Re i c=0 \Longrightarrow \Delta_{(2)} \Re=0 .
$$

This Laplace-like equation for the Riemann tensor appears to have been written down first of all by Penrose [47, motivated by spinors, and later in 44]; in both cases, only four dimensional Ricci-flat spaces were considered. Subsequently the general version (866) has been presented in [5, 48, 46, 9], as a direct result of differentiating the Bianchi equations. The typical index version of (86) reads

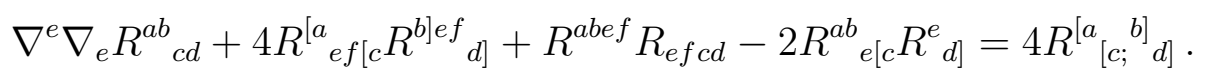

(One should note different sign conventions, and some possible sign inconsistencies in the literature; see [1] for a summary.)

As explained earlier in Section 3, the trace and index symmetry properties across the two pairs of indices do not commute with the operator $\Delta_{(2)}$; and so in particular,

$$
\text { (i) }\left(\Delta_{(2)} \Re\right)_{a[b c d]} \neq 0, \quad \quad(i i) \operatorname{tr}\left(\Delta_{(2)} \Re\right) \neq \Delta_{(2)} \operatorname{tr}(\Re)=\Delta_{(2)} \Re i c
$$

as is easily confirmed. However, this operator $\Delta_{(2)}$ has been proposed in [44, 4, 8, 9] as the natural analogue for the Riemann tensor of the usual de Rham operator $\Delta$ for the electromagnetic field tensor; we would argue, precisely because of these deficiencies, that it is not the most appropriate operator. It should be understood that in these references only vacuum spaces are being explicitly considered, and in such spaces the commutativity properties $(i i)$ which fail in general are satisfied trivially because of the absence of Ricci tensor terms.

Of course, we could have done the whole calculation using the corresponding transposes, and we would easily arrive at

$$
\Delta_{(1)} \Re=-d_{(1)} d_{(2)} \Re i c
$$

which is strictly equivalent to (86), due to the symmetry of the Riemann tensor pairs.

A similar argument could be given concerning the information obtainable using the weighted operator $\bar{\Delta}$. Despite this fact, in general we believe that the operators $\Delta_{(1)}$ and $\Delta_{(2)}$ are potentially confusing and not really particularly suitable; this is especially true for general tensors with no symmetry between blocks, but also for the Riemann tensor itself. We firmly believe that the proper operator to be used here is $\bar{\Delta}$. From (866, 89) it follows immediately that

$$
\bar{\Delta} \Re=-\frac{1}{2}\left(d_{(1)} d_{(2)}+d_{(2)} d_{(1)}\right) \Re i c .
$$


This is a more useful version of a Laplace-like equation for the Riemann tensor, and it does not have the disadvantages of (86). It is easy to check, for instance, that the two examples considered above lead immediately to trivial identities:

$$
\text { (i) }(\bar{\Delta} \Re)_{a[b c d]}=0, \quad \text { (ii) } \operatorname{tr}(\bar{\Delta} \Re)=\bar{\Delta} \operatorname{tr}(\Re)=\bar{\Delta} \Re i c
$$

It should be remarked that the second equation here implies, in fact, that there is no non-trivial expression for $\bar{\Delta} \Re i c$ derivable from (90). If one wishes to find a Laplace-like equation for the Ricci tensor, the best route is to use (37, 38) to take the trace of (83) to get $^{3}$

$$
\delta_{(1)} \Re i c=-\frac{1}{2} d_{(2)} R
$$

where $R=\operatorname{tr}(\Re)=R_{c}^{c}$ is the scalar curvature; from which, together with (85) it follows that

$$
\Delta_{(1)} \Re i c=-\delta_{(1)} \delta_{(2)} \Re-\frac{1}{2} d_{(1)} d_{(2)} R .
$$

For the same reasons as above we prefer to combine it with its $\Delta_{(2)}$ counterpart

$$
\Delta_{(2)} \Re i c=-\delta_{(2)} \delta_{(1)} \Re i c-\frac{1}{2} d_{(2)} d_{(1)} R
$$

to obtain

$$
\bar{\Delta} \Re i c=-\frac{1}{2}\left(\delta_{(1)} \delta_{(2)}+\delta_{(2)} \delta_{(1)}\right) \Re-\frac{1}{4}\left(d_{(1)} d_{(2)}+d_{(2)} d_{(1)}\right) R .
$$

A final contraction here can be obtained by remembering that $\bar{\Delta}$ commutes with contraction, and using (38) on the first term on the right hand side; on the otherhand, we cannot use (37) on the second term since $R$ is a scalar - acting here as a double $(0,0)$-form. However, in index notation $\left[\left(d_{(1)} d_{(2)}+d_{(2)} d_{(1)}\right) R\right]_{a b}=R_{; a b}+R_{; b a}$ and hence its trace is $2 \bar{\Delta} R$. Hence we obtain the usual Laplace-like equation for the scalar curvature $R$

$$
\frac{1}{2} \bar{\Delta} R=-\frac{1}{2}\left(\delta_{(1)} \delta_{(2)}+\delta_{(2)} \delta_{(1)}\right) \Re i c=-\delta_{(1)} \delta_{(2)} \Re i c,
$$

where the last equality is due to the symmetry of the Ricci tensor.

The index version of (90) was written in [18, 1] and reads

$$
\begin{gathered}
\nabla^{e} \nabla_{e} R_{c d}^{a b}+4 R^{[a}{ }_{e f[c} R^{b] e f}{ }_{d]}+R^{a b e f} R_{e f c d}+R^{a b}{ }_{e[c} R_{d]}^{e}+R_{c d}{ }^{e[a} R_{e}{ }^{b]} \\
=2 R^{[a}{ }_{\left[c^{;} d\right]}+{ }^{b}+2 R_{\left[c^{[a} ; d\right]}^{b]}
\end{gathered}
$$

\footnotetext{
${ }^{3}$ This formula (91) is an illustrative example of the possibility mentioned on a number of occasions in this paper that some multiple forms must be considered as having extra blocks with no indices, when combined with other such forms. In this case, despite $R$ being a scalar, one has to take its " $d_{(2)}$ "-derivative. This means that $R$ in this equation acts as a 'double $(0,0)$-form'. The general rule for these situations is to define the form-structure number of an equation as the maximum one for the different terms involved, and then every term in such equation has to be considered accordingly. See also Eqs. (92 951), (71) for other examples.
} 
while the index versions of (94) and (95) are the familiar equations:

$$
\begin{gathered}
\left.\nabla^{e} \nabla_{e} R_{a b}-\frac{1}{2} \nabla_{a} \nabla_{b} R-R_{a}{ }^{c} R_{b c}-R_{a e f b} R^{e f}=\nabla_{e} \nabla_{f} R_{(a}^{e}{ }^{f} b\right) \\
\frac{1}{2} \nabla^{e} \nabla_{e} R=\nabla_{e} \nabla_{f} R^{e f} .
\end{gathered}
$$

We believe that the equation (90) is the natural Riemann tensor analogue of (81), and of course we also have

$$
d_{(1)} \Re i c=0 \Longrightarrow \bar{\Delta} \Re=0 .
$$

Let us comment on the implications of the different definitions of 'harmonic tensors' for the Riemann tensor. From (87), (97) we see that the Riemann tensor is fully harmonic (ergo harmonic) in spaces with $d_{(1)} \Re i c=0\left(\Longleftrightarrow d_{(2)} \Re i c=0\right)$, including manifolds with a parallel Ricci tensor and its particular cases of Ricci-flat and Einstein spaces. As noted above, it has sometimes been the case that the operator $\Delta_{(2)}$ has been considered as the extension to arbitrary tensors of the de Rham operator for single forms; and hence for the special case of the Riemann tensor the condition $\Delta_{(2)} \Re=0$ has been identified as its harmonic condition [4, 9]. However, we want to stress the following two important remarks, which highlight the properties of $\bar{\Delta}$ and support our claim that this is the right operator, giving the proper harmonic condition:

- The class of pseudo-Riemannian manifolds with a harmonic Riemann tensor is larger than that containing a fully harmonic Riemann tensor. This is clear from (86), (89) and (90), as we only need the condition $\left(d_{(1)} d_{(2)}+d_{(2)} d_{(1)}\right) \Re i c=0$ for the former case. (Of course, for compact without boundary proper Riemannian manifolds the fully harmonic and harmonic cases are equivalent, as follows from Theorem 4.3.)

- We also note that the most common Laplacian used for arbitrary tensors, and thought to be mathematically appropriate, has been the Lichnerowicz Laplacian $\Delta_{L}$. We have pointed out, in general, that tensors harmonic in the sense of $\Delta_{L} T=0$, do not coincide with (fully) harmonic tensors; in particular, as follows from (30), the Riemann tensor of pseudo-Riemannian manifolds (compact or not), are not harmonic by using $\Delta_{L}$, not even for Ricci-flat cases. Probably this was the reason that some authors chose equation (866) (or equivalently (89)) to define the Laplacelike or wave equations for the Riemann tensor, letting aside the probably 'more natural' operator $\Delta_{L}$.

We believe that these two remarks taken together with other advantages mentioned before demonstrate that the weighted operator $\bar{\Delta}$ is better suited than either of $\Delta_{(i)}, \Delta_{L}$, and probably the right choice for defining harmonic tensors and studying the derived implications.

Finally, we want to remark about yet another good property of $\bar{\Delta}$ (shared in this case by $\Delta_{(1)}$ and $\left.\Delta_{(2)}\right)$ : for any double $(2,2)$-form $\mathcal{R}$ satisfying the first Bianchi identity (that 
is, a Riemann candidate), its 'Weyl part' is uniquely defined as usual by taking out the traces. We can denote by $W\{\mathcal{R}\}$ the Weyl part of a Riemann candidate. For example, $W\{\Re\}=C$ where $C$ denotes the Weyl tensor. As proved first in [5], the operator $W$ commutes with $\Delta_{(2)}$, and therefore with $\bar{\Delta}$. Hence, this provides the straightest route to the correct Laplace-like equation for the Weyl tensor, because

$$
W\{\bar{\Delta} \Re\}=\bar{\Delta}(W\{\Re\})=\bar{\Delta} C
$$

so that, on using (90) we obtain

$$
\bar{\Delta} C=-\frac{1}{2} W\left\{\left(d_{(1)} d_{(2)}+d_{(2)} d_{(1)}\right) \Re i c\right\} .
$$

Now, it is very easy to produce an index version of this equation. (See [1] for different versions).

\section{Summary and Discussion}

The inspiration for this paper was the result — that there exists a single potential for any Weyl candidate in any dimension - which we obtained in [27] in a rather pragmatic manner; here, that result has been shown to be a special case of a much more general result, which itself is a consequence of a significant generalisation and innovation in formalism. The underlying approach has been the systematic consideration of tensors as $r$-fold forms which has been explained and discussed at length in [49.

The generalisation of the differential form approach to tensor-valued forms and the extension of the use of the exterior differential $d$ to such quantities is well known; we have emphasised the extension of the use of $\delta$ and $\Delta$ also, and highlighted how an $r$ fold form can be thought of as $r$ different tensor-valued forms, by taking each of the $r$ sets of antisymmetric indices as the form indices, and defining the three operators $d_{(i)}, \delta_{(i)}$ and $\Delta_{(i)}, i=1, \ldots r$, associated with each in turn. However, we believe the explicit introduction of the weighted de Rham operator $\bar{\Delta}$ as defined in this paper adds an important new ingredient. Two crucial properties of this operator are that it enables us, via a simple Laplace-like equation, to define an associated superpotential of exactly the same tensor type, and in addition to define potentials in a very natural manner; other generalised Laplacian operators for tensor-valued forms lack one or both of these properties. Hence we believe that $\bar{\Delta}$ is a more powerful operator than $\Delta_{L}$, and so will be very useful in the type of formal investigations where $\Delta_{L}$ has been used previously [41, [42, 7], and as a powerful alternative in investigations of harmonic tensors.

As a matter of fact, we have shown that the harmonicity property derived from the use of $\Delta_{L}$ is different from that derived from $\bar{\Delta}$, and that the latter has better properties concerning the harmonicity of Riemann curvature tensors (Section 7) and in proper Riemannian manifolds (Theorem 4.3). We have also proved that one can use the defin-

ing properties of $\bar{\Delta}$ to define potentials and thereby to construct a generalised Hodge 
decomposition. Theorem 4.2 provides this fundamental generalisation to arbitrary tensors (viewed as $r$-fold forms) of the local Hodge decomposition for antisymmetric tensors (single $p$-forms); an obvious next question to be considered is the possibility of a generalisation of the global Hodge decomposition for closed proper Riemannian manifolds. A first partial step towards this direction has been established in Theorem 4.3, which we believe was not known hitherto.

The remaining task will require, on the one hand, a systematic analysis of the powers of the operators $d_{(i)}$ and $\delta_{(i)}$, and on the other hand, a complete resolution of the relation between tensors with the property $d_{(i)} T=0$, and those with the property $\exists S: T=d_{(i)} S$ (and the dual analogue with $\delta_{(i)}$ instead of $d_{(i)}$.) By the way, this will allow us to see if there are some implications of the Bianchi identities (82 84) for the potentials defined in Theorem 6.3. in the same way as the first of the Maxwell equations (80) implies the existence of a unique electromagnetic potential such that $F=d A$. All these matters are closely related, actually, to the gauge problem for the general potentials that we have mentioned in the paper and is the subject of current investigation [28. We are going to comment along these lines now.

As is well known, the operators $d$ and $\delta$ are clearly nilpotent for single $p$-forms in curved spaces, and for tensor-valued forms in flat spaces. Deeper nilpotent properties for tensor-valued forms in curved space, and deeper commutator relationships with $\bar{\Delta}$, and other operators (such as the first order operator which defines the potential for the Weyl tensor) also need to be explored.

As an application of the general result for all tensors in Theorem 4.2, we considered the Riemann curvature tensor showing that it can be written in terms of a pair of potentials, as given in Theorem 6.3. (There had earlier been some suggestions that the Riemann tensor could be written in terms of one potential via the Riemann-Lanczos equations 10, but subsequently this was shown not to be possible [20, 22] — at least for dimensions $n \geq 3$; the fact that such a single potential cannot exist is now better understood from this new result.) The usefulness of this pair of potentials, and in particular, the implications which the Bianchi equations impose on their relationship, need further study.

Recently there have been attempts in three dimensions to obtain $(2,1)$-form potentials $L_{a b}^{c}$ for the Ricci tensor via the Ricci-Lanczos equations [13,

$$
R_{a b}=L_{a e b}{ }^{i e}+L_{b e a}{ }^{; e}+L_{a e ; b}^{e}+L^{e}{ }_{b e a} .
$$

The reasons for the lack of success in obtaining such potentials for arbitrary Ricci tensors in 13 become apparent when (98) is compared with (71); from the latter it is clear that this type of potential formulation of the Ricci tensor requires two potentials. On the other hand, we know from (172) that the traceless part of the Ricci tensor can always be given in terms of one potential, in arbitrary dimensions; hence the partial success in [13] in investigating special spaces in three dimensions with zero Ricci scalar. Moreover, we can see directly that, by excluding the Ricci scalar from the potential structure, we will avoid the global obstructions involving the Ricci scalar in [13] in all dimensions (and the local obstruction in 22 in three dimensions). It is also instructive to compare the complicated nature of the second order equations for the various superpotentials in [13] 
with the corresponding simple and natural counterpart (40) involving the weighted de Rham operator $\bar{\Delta}$.

There are many interesting aspects of the new potential for the Weyl tensor which require deeper analysis. When the new potential for the Weyl tensor in all dimensions was obtained in [27] we pointed out that, in four dimensions, this new $(2,3)$-form potential $P^{a b}{ }_{c d e}$ is identical to the double dual of the classical Lanczos $(2,1)$-form potential $H_{c}^{a b}$ 40], 38, 24], 2. It is now clear why attempts to find higher dimensional analogues of the Lanczos potential gave negative conclusions [25], 26], 23]; we should have been looking for higher dimensional analogues not of the Lanczos potential, but of its double dual. There do not seem to be deeper implications for this new result in four dimensions; investigations of the Lanczos potential in four dimensions seem to be most efficiently dealt with in spinors 38, 2. However there are very important implications in higher dimensions.

As emphasised in [27], we now have, in all dimensions, an explicit potential for the Weyl tensor which supplies a tensor which is an 'integral' of the Weyl tensor at the level of the connection, and whose 'square' has units $L^{-2}$ which are precisely the units we would expect for gravitational 'energies'. This suggests the usefulness of the wave equation and the super-energy tensor of this potential, in all dimensions with Lorentz signature, as an alternative to the Bel-Robinson tensor for such mathematical investigations as positivity properties, stability and the Cauchy problem for the Einstein equations [12, 3].

We have built our results on second order Laplace-like equations, with appeals to the Cauchy-Kovalewski theorem [15] so that our results are local for analytic pseudoRiemannian metrics. However, we expect that these results can be generalised in the usual way; from the point of view of general relativity we can appeal to stronger theorems 31, 35] when we specialise to spaces with Lorentz signature. However, of course the potentials are by definition first order, and now having established their existence it would be more natural to consider them in a first order system. In particular, the single potential for the Weyl tensor is an attractive candidate for deeper analysis in this context; preliminary investigations indicate that more direct and powerful results can be obtained by treating this definition as part of a first order symmetric hyperbolic system.

When we choose a potential we know that it is not unique, and a full understanding of the role of gauge, which is more complicated for tensor-valued forms than for single $p$-forms, will be very important for further work. In particular we will need to have a set of explicit gauge equations to complete the first order symmetric hyperbolic system for the Weyl tensor. Furthermore, for the Weyl tensor, we would hope for a second-order linear equation for its potential with principal part of type $\nabla^{h} \nabla_{h} P^{a b}{ }_{c d e}$, so that this will give an elliptic equation for positive-definite metrics and a wave equation for Lorentzian signature; in order to obtain such a simple version of the second-order linear equation for the potential we will need to exploit the gauge freedom. In curved four dimensional space (where we can be guided by the Lanczos potential [38, [2], 21]), and in $n$-dimensional flat space it is easy to identify this gauge freedom and obtain the expected simple Laplacelike equation for the potential, but for other dimensions in curved space the calculations become long and complicated [28]. 


\section{Acknowledgements}

JMMS gratefully acknowledges financial support from the Wenner-Gren Foundation, Sweden, and from grants FIS2004-01626 of the Spanish CICyT and no. 9/UPV 00172.31014456/2002 of the University of the Basque Country. JMMS thanks the Matematiska institutionen, Linköpings universitet, where this work was partly carried out, for hospitality.

\section{References}

[1] Andersson, F. and Edgar, S.B. (1996). The wave-equation for the Weyl tensor/spinor and dimensionally dependent tensor identities. Int. J. of Mod. Physics, 5, 217-225.

[2] Andersson, F. and Edgar, S.B. (2001). Existence of Lanczos potentials and superpotentials for the Weyl spinor/tensor. Class. Quantum Gravity, 18, 2297-2304.

[3] Andersson, L. and Moncrief, V. (2003). Elliptic-hyperbolic systems and the Einstein equations. Ann. Henri Poincaré 4, no. 1, 1-34.

[4] Babourova, O.V. and Frolov, B.N. (1995). On a harmonic property of the Einstein manifold curvature. arXiv:gr-qc/9503045

[5] Bel, L. (1963). Étude de certains opérateurs definis sur les formes tensorielles (r,s). Ann. di Mat. Pura ed Applicata, 51, serie 4, 171-192

[6] Benn, I.M. and Tucker, R.W. (1987). An Introduction to Spinors and Geometry with Applications in Physics, Adam Hilger, Institute of Physics.

[7] Besse, A. L. (1987). Einstein Manifolds, Springer-Verlag.

[8] Bini, D., Cherubini, C., Jantzen, R.T., and Rufini, R. (2002). Teukolsky Master Equation: De Rham wave equation for the gravitational and electromagnetic fields in vacuum. Prog. Theor. Phys., 107, 967-992.

[9] Bini, D., Cherubini, C., Jantzen, R.T., and Rufini, R. (2003). De Rham wave equations for tensor valued $p$-forms. Int. J. Mod. Phys. D, 12, 1363-1384.

[10] Brinis Udeschini, E. (1977). Su un tensore triplo potenziale del tensore di Riemann. Mech. Fis. Mat. Istituto Lombardo (rend. Sc.), 111, 466. (1980) A natural scalar field in the Einstein Gravitational Theory. Gen. Rel. Grav., 12, 429.

[11] Cartan, É. (1946). Lecons sur la géométrie des espaces de Riemann, 2nd edn. Gauthier-Villars, Paris. 
[12] Christodoulou, D. and Klainerman, S. (1993). The global nonlinear stability of Minkowski space, Princeton Univ. Press.

[13] Chrúsciel P.T. and Gerber, A. (2005). Some potentials for the curvature tensor on three-dimensional manifolds. Gen. Rel. Grav., 37, 891-905.

[14] Coquereaux, R. (2002). Espaces, Fibrés et Connexions, http://www.cpt.univ-mrs.fr/ coque/book/sourceforhtml.html

[15] Courant, R. and Hilbert, D. (1989). Methods of Mathematical Physics Vol II, Willey Classics Edition.

[16] de Rham, G. (1955). Variétés différentiables, Hermann, Paris.

[17] Deser, S. (1967). Covariant decomposition of symmetric tensors and the gravitational Cauchy problem. Ann. Henri Poincaré Section A, VII, no. 2, 149-188.

[18] de Witt, B. (1962) in Gravitation: An Introduction to Current Research, ed. L. Witten, Witney, New York.

[19] Dubois-Violette, M. and Henneaux, M. (2002). Tensor fields of mixed Young symmetry type and N-complexes. Commun.Math.Phys. 226, 393-418.

[20] Edgar, S. B. (1994). Non-existence of the Lanczos potential for the Riemann tensor in higher dimensions. Gen. Rel. Grav., 26, 329-332.

[21] Edgar, S. B. (1994). The wave equation for the Lanczos tensor/spinor and a new tensor identity. Mod. Phys. Lett. A, 9, 479-482.

[22] Edgar, S. B. (2003). On effective constraints for the Riemann-Lanczos system of equations. J. Math. Phys., 44, 5375-5385.

[23] Edgar, S. B. (2005). Proofs of existence of local potentials for traceless symmetric 2-forms using dimensionally dependent identities. J. Geom. Phys., 54, 251-261.

[24] Edgar, S. B. and Höglund, A. (1997). The Lanczos potential for the Weyl curvature tensor: existence, wave equations and algorithms. Proc. Roy. Soc. A, 453, 835-851.

[25] Edgar, S. B. and Höglund A. (2000). The Lanczos potential for Weyl candidates exists only in four dimensions. Gen. Rel. Grav., 32, 2307-2318.

[26] Edgar, S. B. and Höglund, A. (2002). The non-existence of a Lanczos potential for a Weyl curvature tensor in dimensions $n \geq 7$. Gen. Rel. Grav., 34, 2149-2153.

[27] Edgar, S. B. and Senovilla, J.M.M. (2004). A local potential for the Weyl tensor in all dimensions. Class. Quantum Grav., 21, L133-L137.

[28] Edgar, S. B. and Senovilla, J.M.M. (2005). Gauge freedom for general potentials and new potentials for curvature tensors; in preparation. 
[29] Flanders, H. (1989). Differential Forms with Applications to the Physical Sciences, Dover Publications.

[30] Frankel, T. (1997). The Geometry of Physics, Cambridge University Press.

[31] Friedlander, F. G. (1975). The Wave Equation on a Curved Spacetime, Cambridge University Press.

[32] Gemelli, G. (2000). Second-order covariant tensor decomposition in curved spacetime. Mathematical Physics, Analysis and Geometry, 3, 195-216.

[33] Göckeler, M. and Schücker, T. (1987). Differential geometry, gauge theories, and gravity. Cambridge Monographs on Mathematical Physics, Cambridge University Press.

[34] Goldberg, S.I. (1998 revised ed.) Curvature and Homology, Dover, London

[35] Hawking, S. W. and Ellis, G. F. R. (1973). The large scale structure of spacetime, Cambridge Monographs on Mathematical Physics, No. 1. Cambridge University Press, London-New York.

[36] Helmholtz, H. (1858). Über Integrale der hydrodynamischen Gleichungen, welche den Wirbelbewegungen entsprechen. J. Reine Angew. Math., 55, 25-55

[37] Hodge, W. V. D. (1941) The Theory and Applications of Harmonic Integrals, Cambridge University Press, Cambridge.

[38] Illge, R. (1988). On potentials for several classes of spinor and tensor fields in curved space-times. Gen. Rel. Grav., 20, 551-564.

[39] Kulkarni, R. S. (1972). On the Bianchi Identities. Math. Ann., 199, 175-204.

[40] Lanczos, C. (1962). The splitting of the Riemann tensor. Rev. Mod. Phys., 34, 379389.

[41] Lichnerowicz, A. (1961) Propagateurs et commutateurs en relativité générale, Publ. Scient. des Hautes études scientifiques, No.10, Hermann, Paris.

[42] Lichnerowicz, A. (1964). Propagateurs, commutateurs, et anticommutateurs en relativité générale, in "Relativity, Groups and Topology", eds. C. DeWitt and B.S. DeWitt, Gordon and Breach, New York.

[43] Lindell, L. V. and Dassios, G. (2003). Helmoltz Theorem for multiform fields. J. Electromagn. Waves and Appl., 17, 3-14.

[44] Misner, C.W., Thorne, K. S. and Wheeler, J.A. (1973). Gravitation, Freeman.

[45] Nakahara, M. (1990). Geometry, Topology and Physics, Institute of Rhysics. 
[46] Parker, L. and Christensen, S.M. (1994). MathTensor, Addison-Wesley, New York.

[47] Penrose, R. (1960). Ann. Phys., 10, 171-201.

[48] Ryan, M.P. (1974). Teukolsky equation and Penrose wave equation. Phys. Rev., D, 10, 1736-1740.

[49] Senovilla, J. M. M. (2000). Super-energy Tensors. Class. Quantum Grav., 17, 27992841.

[50] Schwarz, G. (1991). Hodge Decomposition — a Method for Solving Boundary value Problems, Springer-Verlag.

[51] Thirring, W. (1978). Classical Field Theory, Springer-Verlag.

[52] Urakawa, H. (1993). Calculus of Variations and Harmonic Maps, A.M.S..

[53] Woodside, D. A. (1999). Uniqueness theorems for classical four-vector fields in Euclidean and Minkowski spaces. J. Math. Phys., 40, 4911-4943.

[54] York, J.W. (1973). Conformally invariant orthogonal decomposition of symmetric tensors on Riemannian manifolds and the initial-value problem of general relativity. J. Math. Phys., 14, 456-471. 